\title{
An Innovative Approach to Characterize Clinical ADME and Pharmacokinetics of the Inhaled Drug Nemiralisib Using an Intravenous Microtracer Combined with an Inhaled Dose and an Oral Radiolabel Dose in Healthy Male Subjects ${ }^{\mathbb{S}}$
}

\author{
Andrew W. Harrell, Robert Wilson, Yau Lun Man, Kylie Riddell, Emily Jarvis, Graeme Young, \\ Robert Chambers, Lee Crossman, Alex Georgiou, Adrian Pereira, David Kenworthy, \\ Claire Beaumont, Miriam Marotti, Denisa Wilkes, Edith M. Hessel, and William A. Fahy
}

Drug Metabolism and Pharmacokinetics (A.W.H., G.Y., R.C., D.K.) and Bioanalysis, Immunogenicity and Biomarkers (A.G., A.P.), GlaxoSmithKline R\&D, Ware, United Kingdom; RD Projects Clinical Platforms \& Sciences (R.W.), Drug Metabolism and

Pharmacokinetics (C.B.), Discovery Medicine (Y.L.M.), Biostatistics (E.J.), GlaxoSmithKline R\&D and Refractory Respiratory Inflammation Discovery Performance Unit, GlaxoSmithKline, Stevenage, United Kingdom (E.M.H.); Safety and Medical Governance (M.M.) and Discovery Medicine (W.A.F.), GlaxoSmithKline R\&D, Stockley Park, Uxbridge, United Kingdom; Global Clinical and Data

Operations, GlaxoSmithKline R\&D, Ermington, Australia (K.R.); Covance Laboratories, Harrogate, United Kingdom (L.C.); and Hammersmith Medicines Research, London, United Kingdom (D.W.)

Received October 10, 2019; accepted October 12, 2019

\begin{abstract}
An innovative open-label, crossover clinical study was used to investigate the excretion balance, pharmacokinetics, and metabolism of nemiralisib-an inhaled phosphoinositide 3-kinase delta inhibitor being developed for respiratory diseases. Six healthy men received a single intravenous microtracer of $10 \mu \mathrm{g}\left[{ }^{14} \mathrm{C}\right]$ nemiralisib with a concomitant inhaled nonradiolabeled $1000 \mu \mathrm{g}$ dose followed by an oral $800 \mu \mathrm{g}$ dose of $\left[{ }^{14} \mathrm{C}\right]$ nemiralisib 14 days later. Complementary methods including accelerator mass spectrometry allowed characterization of a range of parameters including oral absorption $\left(F_{\text {abs }}\right)$, proportion of nemiralisib escaping gut wall metabolism $\left(F_{g}\right)$, hepatic extraction $\left(E_{h}\right)$, fraction of dose absorbed from inhaled dose ( $\left.F_{\text {lung }}\right)$, and renal clearance. Intravenous pharmacokinetics of nemiralisib were characterized by low blood clearance $(10.0 \mathrm{l} / \mathrm{h})$, long terminal half-life ( 55 hours), and high volume of distribution at steady state (728 I). Nemiralisib exhibited moderate inhaled and oral bioavailability ( $38 \%$ and $35 \%$ ) while $F_{\text {lung }}$ was $29 \%$. Absorption and first-pass parameters were corrected for blood renal clearance and compared with values without correction. Any swallowed nemiralisib was relatively well absorbed $\left(F_{\text {abs }}, 0.48\right)$ with a high fraction
\end{abstract}

escaping gut wall metabolism and low extraction by the liver $\left(F_{g}\right.$ and $E_{h}$ being 0.83 and 0.10 , respectively). There were no major human plasma metabolites requiring further qualification in animal studies. Both unchanged nemiralisib and its oxidative/conjugative metabolites were secreted in bile, with nemiralisib likely subject to further metabolism through enterohepatic recirculation. Direct renal clearance and metabolism followed by renal clearance were lesser routes of elimination.

\section{SIGNIFICANCE STATEMENT}

A number of innovative features have been combined into one small clinical study enabling a comprehensive description of the human pharmacokinetics and metabolism of an inhaled molecule. Design elements included an intravenous ${ }^{14} \mathrm{C}$ tracer administration concomitant with an inhalation dose that enabled derivation of parameters such as fraction absorbed $\left(F_{\text {abs }}\right)$, the proportion of drug escaping first-pass extraction through the gut wall and liver $\left(F_{g}\right.$ and $\left.F_{h}\right)$ and hepatic extraction $\left(E_{h}\right)$. Entero-test bile sampling enabled characterization of biliary elimination pathways.
Introduction

Nemiralisib (bis(6-(1H-indol-4-yl)-4-(5-\{[4-(propan-2-yl)piperazin1 -yl]methyl $\}-1,3$-oxazol-2-yl)- $1 H$-indazole) succinate) is a potent and

This study was funded by GlaxoSmithKline.

A.W.H., R.W., Y.L.M., E.J., G.Y., R.C., A.G., A.P., D.K., C.B., W.A.F. are GSK employees and hold GSK shares. K.R., M.M. are GSK employees. E.M.H. is a GSK employee and holds GSK shares and is on patents pertaining to the research discussed in the manuscript. L.C., D.W. have no conflicts of interest to disclose. Availability of data and materials.

Information on GSK's data sharing commitments and requesting access can be found at: https://www.clinicalstudydatarequest.com.

https://doi.org/10.1124/dmd.119.088344.

$\$$ This article has supplemental material available at dmd.aspetjournals.org. selective inhaled inhibitor of phosphoinositide 3-kinase delta being developed for the treatment of chronic respiratory disorders (Down et al., 2015; Cahn et al., 2017; Begg et al., 2019). The characterization of human absorption, distribution, metabolism, and excretion (referred to as $\mathrm{ADME}$ ) and pharmacokinetics (PK) is an important and required part of drug development. Human ADME qualifies whether the toxicology species are relevant to the safety assessment of the drug for human use and informs on the utility of specific clinical safety studies including investigations of possible drug interactions or special patient groups (e.g., hepatic or renal impaired patients). Intravenous PK is needed to characterize primary PK parameters such as bioavailability, clearance and volume of distribution, which contribute to a fundamental understanding of how the drug enters, distributes, and leaves the body. A full ADME investigation usually includes administration of a radioisotope 
using the dose, route, and formulation of interest. This presents many challenges for those drugs intended to be administered by extravascular routes such as inhaled nemiralisib.

The complications associated with administering a radioisotope by inhalation are not trivial (Harrell et al., 2013). Although precedented (Affrime et al., 2000), it is challenging and often not possible to recreate the exact intended commercial form, including particle size in the intended clinical device. Other complications associated with running an inhaled human ADME study include not being able to quantify accurately the dose crucial for a full interpretation of the ADME data. For this reason, the human metabolism of nemiralisib was characterized using an intravenous microtracer administration as a surrogate for the proportion of the inhaled dose reaching the systemic circulation through the lung, while the swallowed portion was characterized through oral administration of $\left[{ }^{14} \mathrm{C}\right]$ nemiralisib (in a separate dosing period).

Traditionally, intravenous $\mathrm{PK}$ is defined following a two-way crossover design in which healthy subjects receive single intravenous and extravascular therapeutically relevant administrations on separate occasions. A drawback of this approach is that the therapeutic intravenous dose requires an intravenous toxicology package to support the clinical trials application. To encourage wider application of exploratory clinical studies, harmonized International Committee on Harmonization guidance was developed to allow ultra-low intravenous administration $(<100 \mu \mathrm{g})$ to humans with a substantially reduced toxicology package (https://www.ich. org/fileadmin/PublicWebSite/ICHProducts/Guidelines/Multidisciplinary/ M3R2/Step4/M3R2Guideline.pdfhttps://database.ich.org/sites/default/ files/M3_R2_Guideline.pdf). Referred to as microdosing, the approach commonly involves the incorporation of a ${ }^{14} \mathrm{C}$-isotope into the drug so that systemic exposure can be measured by highly sensitive analytical technologies such as accelerator mass spectrometry (AMS). A further development of the microdose design is to administer the ${ }^{14} \mathrm{C}$-labeled microdose concomitantly with an extravascular dose in a so-called "microtracer" design, where intravenous PK can be defined at therapeutically relevant concentrations (Lappin, 2016). Herein, we develop the approach described by Ambery et al. (2018) for inhaled batefenterol, whereby an intravenous ${ }^{14} \mathrm{C}$-tracer dose of nemiralisib was administered concomitantly with a therapeutically relevant inhaled dose delivered using the intended commercial formulation and device.

Human biliary disposition can be investigated by noninvasive collection of human duodenal bile samples using the Entero-Test device (HDC Corp., Mountain View, CA) (Bloomer et al., 2013). Duodenal bile sampling presents many advantages over stool sampling to provide understanding of biliary secretion of parent drug and metabolites. For example, there is no need to extract drug-related material from complex fecal matter, the resultant metabolic profile is a better indicator of drug and metabolites secreted in bile and any consideration of metabolism of the drug by gut micro flora becomes unnecessary. To take full advantage of the intravenous ${ }^{14} \mathrm{C}$ microtracer dose, the Entero-Test was deployed to fully characterize nemiralisib metabolites secreted in bile as well as provide an indication of possible enterohepatic recirculation.

It is common industry practice to estimate oral absorption $\left(\mathrm{F}_{\mathrm{abs}}\right)$ from human radiolabel studies where an intravenous dose has been included and at least three different approaches can be used (Table 1). Less commonly, further derivations of these equations can be used to describe other first-pass parameters such as the proportion of nemiralisib escaping first-pass extraction through the gut wall and liver $\left(\mathrm{F}_{\mathrm{g}}\right.$ and $\left.\mathrm{F}_{\mathrm{h}}\right)$ and hepatic extraction $\left(\mathrm{E}_{\mathrm{h}}\right)$, which, to the authors knowledge, have never been published in full within the context of a human ADME study. Herein, we review equations for the derivation of absorption and firstpass parameters and apply them to nemiralisib.

Our study includes a number of innovative approaches combined here for the first time into a single clinical study, which aimed to fully characterize and understand the human ADME and PK profile of inhaled nemiralisib.

\section{Materials and Methods}

Nemiralisib, $\left[{ }^{14} \mathrm{C}\right]$ nemiralisib, and $\left[{ }^{2} \mathrm{H}_{7}\right]$ nemiralisib were supplied by Chemical Development, GlaxoSmithKline (GSK) R\&D, Stevenage, UK. All other solvents and reagents were of analytical grade and were purchased from commercial suppliers. In all cases, nemiralisib hemisuccinate salt was administered to study participants. All dose levels herein are calculated in terms of nemiralisib free base.

\section{Study Design}

This was an open-label, single center, nonrandomized, 2-period, singlesequence crossover study in healthy men conducted at Hammersmith Medicines Research Center (London, UK) between November 2017 and December 2017 (GSK study number 206764; ClinicalTrials.gov NCT03315559). The study adhered to the Declaration of Helsinki, was approved by the London Harrow Research Ethics Committee, and each subject gave their written informed consent prior to any study procedure.

Following a screening visit, subjects underwent two treatment periods, conducted on-site, with a 14-day washout between treatments (Fig. 1). In treatment period 1, subjects inhaled $1000 \mu \mathrm{g}$ free base of nonradiolabeled nemiralisib as a dry powder from the ELLIPTA inhaler device (GSK, Brentford, UK). Nemiralisib hemisuccinate salt was blended with lactose and magnesium stearate and packed in foil blisters containing $500 \mu \mathrm{g}$ free base per blister, requiring two inhalation actuations to achieve the full $1000 \mu \mathrm{g}$ dose. Within 5 minutes, each subject received $10 \mu \mathrm{g}\left[{ }^{14} \mathrm{C}\right]$ nemiralisib free base (approximately $22.2 \mathrm{kBq} ; 0.6 \mu \mathrm{Ci}$ ) via intravenous microtracer infusion over 15 minutes. $\left[{ }^{14} \mathrm{C}\right]$ Nemiralisib hemisuccinate salt was dissolved in normal saline at $1 \mu \mathrm{g} / \mathrm{ml}$ so that $10 \mu \mathrm{g}$ free base was administered in a volume of $10 \mathrm{ml}$. Blood, urine, and fecal samples were collected up to 168 hours ( 7 days) after dosing. In treatment period 2, subjects were administered $800 \mu \mathrm{g}$ free base of $\left[{ }^{14} \mathrm{C}\right]$ nemiralisib hemisuccinate salt $(1850 \mathrm{kBq} ; 50 \mu \mathrm{Ci})$ as an oral solution in water $\left(800 \mu \mathrm{g}\left[{ }^{14} \mathrm{C}\right]\right.$ nemiralisib free base was dissolved in $10 \mathrm{ml}$ water). Samples were collected for a minimum of 168 hours and up to 336 hours (day 15 ) or until the amount of radioactivity excreted by each subject was $<1 \%$ per day of the administered dose on two consecutive 24-hour excreta collection intervals. Each subject was exposed to an effective radioactive dose of 0.75 $\mathrm{mSv}$, well within the safety limits defined by the World Health Organization category II $(0.5-5 \mathrm{mSv}$; within the dose limits for members of the public) and the International Commission on Radiologic Protection category IIa (0.1-1 mSv; minor risk) (ICRP, 1991).

\section{Study Population}

Healthy men were aged from 30 to 55 years, weighed $\geq 50 \mathrm{~kg}$ with a body mass index of $19-31 \mathrm{~kg} / \mathrm{m}^{2}$, and had a history of regular bowel movements. Subjects had no history of drug or alcohol abuse, no clinically relevant disease, were nonsmokers, had not taken other medications within the last 7 days (14 days if a potential enzyme inducer), and had not been exposed to significant radiation within the last 12 months. Full details of the eligibility criteria can be found on ClinicalTrials.gov; NCT03315559.

ABBREVIATIONS: ADME, absorption, distribution, metabolism and excretion; Ae, amount excreted; AMS, accelerator mass spectrometry; $\mathrm{CL}_{\mathrm{r}}$, renal clearance; $E_{h}$, hepatic extraction; $F_{a b s}$, fraction absorbed following oral dose; $F_{g}$, fraction escaping gut wall metabolism; $F_{h}$, fraction escaping hepatic metabolism; $F_{\text {lung }}$, fraction of dose absorbed from inhaled dose; $F_{\text {oral }}$, oral bioavailability; LLQ, lower limit of quantification; LSC, liquid scintillation countingpg Eq/mL, picogram equivalents (radioactivity) per mL; PK, pharmacokinetics; R\&D, Research \& Development; $t_{1 / 2}$, terminal phase half-life; $t_{\max }$, time of maximum observed concentration; UPLC, ultra-performance liquid chromatography. 
TABLE 1

Equations to calculate absorption and first-pass pharmacokinetic parameters for nemiralisib

Equation 1 is a commonly used approach to estimate oral absorption $\left(\mathrm{F}_{\mathrm{abs}}\right)$ whereby the \% radioactive dose excreted in urine is compared with oral and intravenous administrations. This approach is

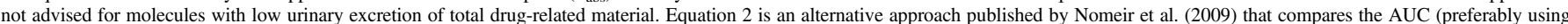

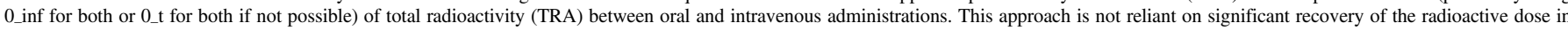

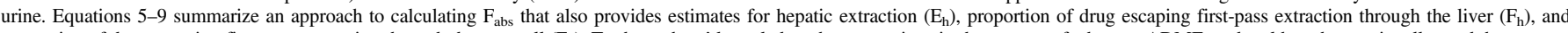

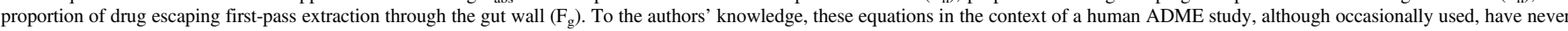

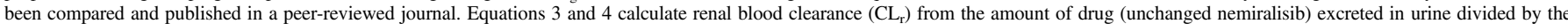

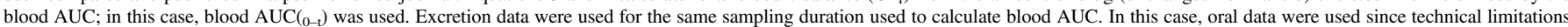

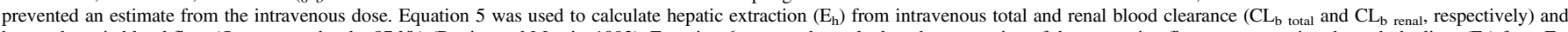

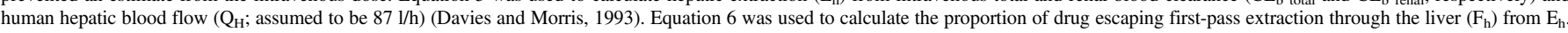

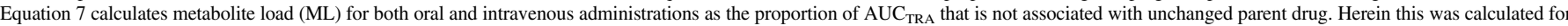

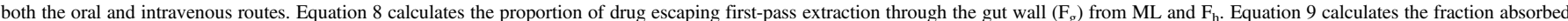
$\left(\mathrm{F}_{\mathrm{abs}}\right)$ from $\mathrm{F}_{\mathrm{h}}$, oral bioavailability $\left(\mathrm{F}_{\text {oral }}\right)$ and $\mathrm{F}_{\mathrm{g}}$. All equations were applied to clinical study data outside the original study plan or post hoc.

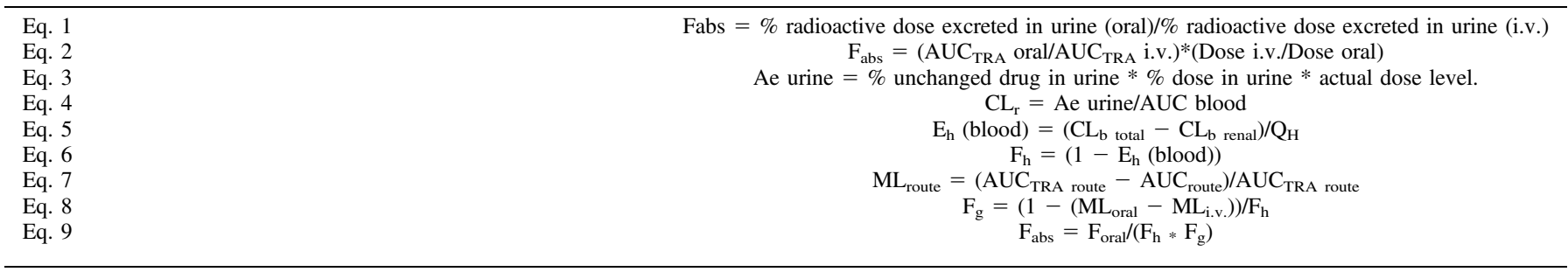

\section{Sample Collection and Processing}

Blood samples were collected up to 7 days after dosing into ethylenediaminetetraacetic acid tubes. Blood volumes were $3 \mathrm{ml}$ for all pharmacokinetic sampling times with an additional $9 \mathrm{ml}$ collected for metabolite sampling at specified sample times $(0.5$ and 4 hours after dosing for period 1 or 2,6 , and 24 hours after dosing for period 2). Plasma was harvested from blood following centrifugation. Urine and fecal samples were collected for 7 days in treatment period 1 and for between 7 and 15 days or until $<1 \%$ radioactive dose was recovered in any 24-hour period in treatment period 2. Sampling of duodenal bile during treatment period 1 was conducted using the Entero-Test string device (Guiney et al., 2011), which was swallowed approximately 3.5 hours before the inhaled dose and removed about 2.5 hours after the end of the infusion (2.75 hours post infusion start). Approximately 2 hours before removal, a food cue was used to stimulate gall bladder emptying. The food cue involved providing visual images of food and asking the participants to imagine eating food, providing a sniff of orange zest and a high fat food morsel (e.g., sausage sandwich). Samples of urine, feces, and bile were stored frozen prior to shipment for analysis.

\section{Sample Analysis}

Mass Balance and Excretion. Total radioactivity excreted in urine and fecal samples was determined using AMS in samples collected during treatment period 1 (Xceleron Inc., Germantown, Maryland, USA) and by liquid scintillation counting (LSC) in samples collected during treatment period 2 (Covance Laboratories Limited, Harrogate, UK). Where levels of radioactivity were too low to be detected by LSC, samples were analyzed by AMS. No cross validation was performed between LSC and AMS because substantial literature supports the individual qualification of both techniques in isolation (Garner et al., 2000; Keck

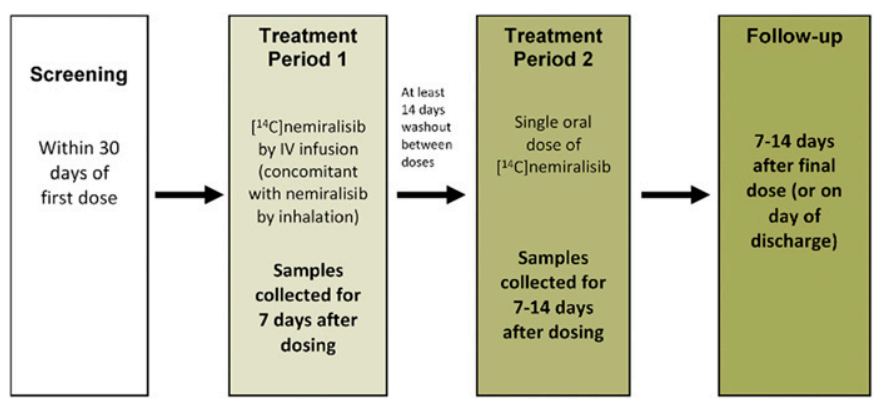

Fig. 1. Study schematic In both treatment periods, treatments were administered after overnight fasting ( $\geq 8$ hours). If radioactivity was higher than $1 \%$ upon discharge, fecal samples were collected by subjects at home after day 15 . et al., 2010). For LSC, efficiency correlation curves were routinely checked through the use of ${ }^{14} \mathrm{C}$ quenched standards. AMS instrument standards (independently internationally established by consensus) were analyzed along with process standards for the graphitization process. Urinary radioactivity was measured in triplicate weighed aliquots by LSC using a Packard TriCarb liquid scintillation counter (Canberra Packard, Pangbourne, Berkshire, UK). Feces samples were combusted using a Perkin Elmer (Pangbourne, Berkshire, UK) Sample Oxidizer. The combusted products were absorbed in Carbosorb (Perkin Elmer Life Sciences, Ltd.) and mixed with Permafluor E+ scintillation fluid (Perkin Elmer Life Sciences, Ltd.). The efficiency of oxidation was determined by combustion of quality control standards, but no corrections were made for combustion efficiency. For each individual run to be acceptable, the efficiency of oxidation measured at the beginning and end of each run was required to be $95 \%-105 \%$. The lower limits of quantification (LLQ) were assigned as twice the mean background disintegrations.

For measurement of total radioactivity by AMS, urine and feces samples underwent combustion (oxidation) and graphitization (reduction) and radioactivity levels were determined using a Single Stage AMS 250KV system (NEC, Middleton, WI) (Young et al., 2008). The mean LLQ was $0.15 \mathrm{pg}$ nemiralisib Eq/ml for urine measurements and $0.954 \mathrm{pg} \mathrm{Eq} / \mathrm{g}$ in feces samples.

Pharmacokinetic Assessments. Plasma samples were analyzed for nemiralisib concentrations (Aptuit, Verona, Italy) following inhaled and oral administration of nemiralisib (periods 1 and 2) using a validated analytical method based on protein precipitation with acetonitrile containing $\left[{ }^{2} \mathrm{H}_{7}\right]$ nemiralisib as an internal standard, followed by liquid chromatography and TurboIonSpray tandem mass spectrometric detection analysis). The LLQ was $20 \mathrm{pg} / \mathrm{ml}$ using a $50 \mu \mathrm{l}$ aliquot of human plasma with a higher limit of quantification of $10,000 \mathrm{pg} / \mathrm{ml}$. Typically, a $3 \mu \mathrm{l}$ aliquot of extracted sample was injected onto a liquid chromatography system consisting of a $50 \times 2.1 \mathrm{~mm}$ internal diameter, Agilent Zorbax SB-CN $5 \mu \mathrm{m}$ column and a Waters Acquity UPLC chromatography system. Mobile phases of $10 \mathrm{mM}$ ammonium formate and acetonitrile at a flow rate of $0.8 \mathrm{ml} / \mathrm{min}$ and a column temperature of $40^{\circ} \mathrm{C}$ were used to elute nemiralisib at a typical retention time of 0.9 minutes. Concentration data generated from treatment period 1 were deconvoluted to derive drug concentrations following inhalation administration by subtraction of drug concentrations determined by AMS (referred to as $\left[{ }^{14} \mathrm{C}\right]$ nemiralisib concentration) from the intravenous microdose administration. Plasma total radioactivity and $\left[{ }^{14} \mathrm{C}\right]$ nemiralisib were analyzed by the Department of Bioanalysis, GSK, Ware, UK. Plasma total radioactivity was assessed by direct AMS for period 1 samples (inhaled + intravenous dose), which had an LLQ of $0.629 \mathrm{pg}$ Eq/ml. For period 2 plasma samples (oral dose), total radioactivity was assessed by LSC, which had an LLQ of $49.0 \mathrm{pg} \mathrm{Eq} / \mathrm{ml}$. Plasma $\left[{ }^{14} \mathrm{C}\right]$ nemiralisib (parent drug following intravenous microdose administration) was determined using a validated analytical method based on protein precipitation, followed by ultra-high-performance 
TABLE 2

Summary of PK parameters following $10 \mu \mathrm{g}$ i.v. $\left[{ }^{14} \mathrm{C}\right]$ nemiralisib $+1000 \mu \mathrm{g}$ inhaled nemiralisib and $800 \mu \mathrm{g}$ oral $\left[{ }^{14} \mathrm{C}\right] \mathrm{nemiralisib}$

All parameters calculated from individual data as presented in Supplemental Table 1.

\begin{tabular}{|c|c|c|c|c|c|}
\hline PK Parameter (unit) & $N$ & $n$ & Route of Administration & Geometric Mean $(\mathrm{CVb} \%)$ & $95 \%$ Confidence Interval \\
\hline \multicolumn{6}{|c|}{ Total radioactivity following $10 \mu \mathrm{g}$ i.v. $\left[{ }^{14} \mathrm{C}\right]$ nemiralisib $+1000 \mu \mathrm{g}$ inhaled nemiralisib and $800 \mu \mathrm{g}$ oral $\left[{ }^{14} \mathrm{C}\right]$ nemiralisib } \\
\hline \multirow{2}{*}{$\mathrm{C}_{\max }(\mathrm{pq} / \mathrm{ml})$} & 6 & 6 & i.v. & $204(52.3)$ & $(122,343)$ \\
\hline & 6 & 6 & Oral & $704(24.4)$ & $(546,906)$ \\
\hline \multirow{2}{*}{$\mathrm{t}_{\max }{ }^{a}(\mathrm{~h})$} & 6 & 6 & i.v. & $0.233(0.233,0.233)$ & NA \\
\hline & 6 & 6 & Oral & $7.00(2.00,8.02)$ & NA \\
\hline \multirow{2}{*}{$\mathrm{AUC}_{0-\mathrm{t}}^{b}\left(\mathrm{~h}^{*} \mathrm{pg} \mathrm{Eq} / \mathrm{ml}\right)$} & 6 & 6 & i.v. & $770(17.7)$ & $(641,926)$ \\
\hline & 6 & 6 & Oral & $31,730(35.7)$ & $(22,057,45,644)$ \\
\hline \multirow{2}{*}{$\mathrm{AUC}_{0-\mathrm{inf}}\left(\mathrm{h}^{*} \mathrm{pg} \mathrm{Eq} / \mathrm{ml}\right)$} & 6 & 3 & i.v. & $813(8.18)$ & $(664,996)$ \\
\hline & 6 & 1 & Oral & 34,749 (NE) & NE \\
\hline \multirow[b]{2}{*}{$t_{1 / 2}(\mathrm{~h})$} & 6 & 3 & i.v. & $56.9(7.70)$ & $(47.0,68.9)$ \\
\hline & 6 & 1 & Oral & $39.6(\mathrm{NE})$ & $\mathrm{NE}$ \\
\hline 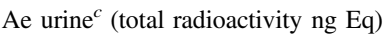 & 6 & 6 & i.v. & 1044 (NA) & NA \\
\hline \multirow{2}{*}{\multicolumn{6}{|c|}{ Intravenous $\left[{ }^{14} \mathrm{C}\right]$ nemiralisib }} \\
\hline & & & & & \\
\hline Radiometric dose $(\mu \mathrm{g})$ & 6 & 6 & i.v. & $8.10(3.2)$ & $(7.83,8.37)$ \\
\hline$C_{\max }(\mathrm{pg} / \mathrm{ml})$ & 6 & 6 & i.v. & $206(57.9)$ & $(117,362)$ \\
\hline$t_{\max }{ }^{a}(\mathrm{~h})$ & 6 & 6 & i.v. & $0.233(0.233,0.233)$ & NA \\
\hline $\mathrm{AUC}_{0-\mathrm{t}}{ }^{d}\left(\mathrm{~h}^{*} \mathrm{pg} / \mathrm{ml}\right)$ & 6 & 6 & i.v. & $546(45.3)$ & $(347,860)$ \\
\hline $\mathrm{AUC}_{0-\text { inf }}\left(\mathrm{h}^{*} \mathrm{pg} / \mathrm{ml}\right)$ & 6 & 4 & i.v. & $751(48.3)$ & $(362,1555)$ \\
\hline $\mathrm{AUC}_{0-t}$ ratio & 6 & 6 & i.v. & $0.709(29.2)$ & $(0.525,0.958)$ \\
\hline $\mathrm{AUC}_{0-\text { inf }}$ ratio & 6 & 2 & i.v. & $0.722(50.3)$ & $(0.010,51.406)$ \\
\hline$t_{1 / 2}(\mathrm{~h})$ & 6 & 4 & i.v. & $54.7(36.0)$ & $(31.4,95.4)$ \\
\hline $\mathrm{CL}^{e}(\mathrm{l} / \mathrm{h})$ & 6 & 4 & i.v. & $10.8(46.7)$ & $(5.32,21.9)$ \\
\hline $\mathrm{Vz}(1)$ & 6 & 4 & i.v. & $851(14.0)$ & $(682,1062)$ \\
\hline Vss (1) & 6 & 4 & i.v. & $728(11.2)$ & $(609,870)$ \\
\hline \multicolumn{6}{|l|}{ Inhaled nemiralisib } \\
\hline Nominal dose $(\mu \mathrm{g})$ & 6 & 6 & Inhaled & $1000(0)$ & NA \\
\hline$C_{\max }(\mathrm{pg} / \mathrm{ml})$ & 6 & 6 & Inhaled & $5259(62.8)$ & $(2871,9633)$ \\
\hline$t_{\max }^{a}(\mathrm{~h})$ & 6 & 6 & Inhaled & $0.0333(0.017,0.050)$ & NA \\
\hline $\mathrm{AUC}_{0-\mathrm{t}}{ }^{f}\left(\mathrm{~h}^{*} \mathrm{pg} / \mathrm{ml}\right)$ & 6 & 6 & Inhaled & $28,426(34.5)$ & $(19,998,40,406)$ \\
\hline $\mathrm{AUC}_{0-\inf }\left(\mathrm{h}^{*} \mathrm{pg} / \mathrm{ml}\right)$ & 6 & 6 & Inhaled & $33,374(33.2)$ & $(23,773,46,854)$ \\
\hline$t_{1 / 2}(\mathrm{~h})$ & 6 & 6 & Inhaled & $58.4(16.8)$ & $(49.0,69.7)$ \\
\hline \multicolumn{6}{|l|}{ Oral nemiralisib } \\
\hline Radiometric dose $(\mu \mathrm{g})$ & 6 & 6 & Oral & $769(0.65)$ & $(763,774)$ \\
\hline$C_{\max }(\mathrm{pg} / \mathrm{ml})$ & 6 & 6 & Oral & $399(27.8)$ & $(300,531)$ \\
\hline$t_{\max }^{a}(\mathrm{~h})$ & 6 & 6 & Oral & $6.00(2.00,8.00)$ & NA \\
\hline $\mathrm{AUC}_{0-t}{ }^{g}\left(\mathrm{~h}^{*} \mathrm{pg} / \mathrm{ml}\right)$ & 6 & 6 & Oral & $16,913(53.8)$ & $(9960,28,719)$ \\
\hline $\mathrm{AUC}_{0-\text { inf }}\left(\mathrm{h}^{*} \mathrm{pg} / \mathrm{ml}\right)$ & 6 & 4 & Oral & $25,108(46.3)$ & $(12,451,50,632)$ \\
\hline $\mathrm{AUC}_{0-t}$ ratio & 6 & 6 & Oral & $0.533(24.7)$ & $(0.413,0.688)$ \\
\hline $\mathrm{AUC}_{0-\text { inf }}$ ratio & 6 & 1 & Oral & $0.377(\mathrm{NE})$ & NE \\
\hline$t_{1 / 2}(\mathrm{~h})$ & 6 & 4 & Oral & $50.8(40.2)$ & $(27.5,94.0)$ \\
\hline CLr (1/h) (parent nemiralisib) & 6 & 6 & Oral & 0.93 (from pooled radioprofiling) & $\mathrm{NE}$ \\
\hline
\end{tabular}

$N$, number of subjects; $n$, number of evaluable subjects; NA, not applicable; NE, not estimable; Vz, volume of distribution during terminal phase after intravenous administration.Vss, volume of distribution at steady state

${ }^{a} t_{\max }$ expressed as median and range.

${ }^{b} \mathrm{AUC}_{0-t}=168 \mathrm{~h}$ for intravenous and $96-168 \mathrm{~h}$ for oral.

${ }^{c} \mathrm{Ae}$ urine, total radioactivity in urine sample $(0-168 \mathrm{~h})$ shown as arithmetic mean.

${ }^{d} \mathrm{AUC}_{0-t}=72-168 \mathrm{~h}$.

${ }^{e} 10.8 \mathrm{l} / \mathrm{h}$ based on plasma or $(10.8 \mathrm{l} / \mathrm{h} / 1.08$ ratio) $10.0 \mathrm{l} / \mathrm{h}$ for blood, i.e., corrected for blood:plasma ratio of 1.08 (unpublished GSK data).

${ }^{f} \mathrm{AUC}_{0-t}=96-168 \mathrm{~h}$

${ }^{g} \mathrm{AUC}_{0-t}=72-168 \mathrm{~h}$.

liquid chromatography + AMS. The LLQ of this method was $1.00 \mathrm{pg} / \mathrm{ml}$ using a $100 \mu \mathrm{l}$ aliquot of plasma. Further details of the analytical methodologies are provided in the online Supplemental Methods.

Quantification and Characterization of Metabolites. Using samples taken following both dosing periods, an equal volume of plasma from each subject was pooled to produce a single representative plasma sample per time point for each dosing route. With these sample pools, a single plasma pool (representative of the area under the plasma concentration time curve from time 0 to 24 hour) was prepared for each dosing route by combining volumes in proportion to the time interval between individual samples (Hop et al., 1998). For each dosing group, a pooled urine sample was prepared for individual subjects by taking an equal percentage by weight $(0.1 \%)$ from each time point cumulatively containing approximately $90 \%$ of the total radioactive drug-related material excreted by this route. These individual pools were then used to prepare a single urine pool per dose route by pooling equal volumes. Individual pooled fecal homogenate samples were prepared from both dosing periods using a similar approach. Here, individual pools were prepared by taking $1 \%$ by weight from each time-point until the pool cumulatively contained approximately $\geq 90 \%$ of the excreted radioactivity for that matrix per subject. A combined fecal pool was then prepared by taking an equal weight from each individual pool. Plasma and feces samples were extracted with acetonitrile, dried using a centrifugal evaporator and reconstituted in 50/50, acetonitrile/water $(\mathrm{v} / \mathrm{v})$. Duodenal bile was extracted with acetonitrile from the Entero-Test bile strings (collected following inhaled + intravenous microtracer dosing) and the extracts were mixed with scintillation fluid prior to counting. LSC vials were then individually counted for 15 minutes duration using a Packard Tricarb low level scintillation counter. Bile string extracts from the two subjects containing the highest levels of radioactivity were pooled to determine the metabolic profile. Metabolites were identified and quantified (where possible) in plasma, urine, feces and bile string extract samples using off-line radio-UPLC with either Topcount (feces sample extracts) or AMS (plasma, urine, and bile sample extracts) as off-line radio-detector. Off-line radiodetection by Topcount was conducted by collecting fractions into 96 shallow well Luma microtiter plates (Perkin Elmer) containing solid scintillant. The liquid chromatography eluate fractions were allowed to evaporate (in air at room temperature) and the dried 


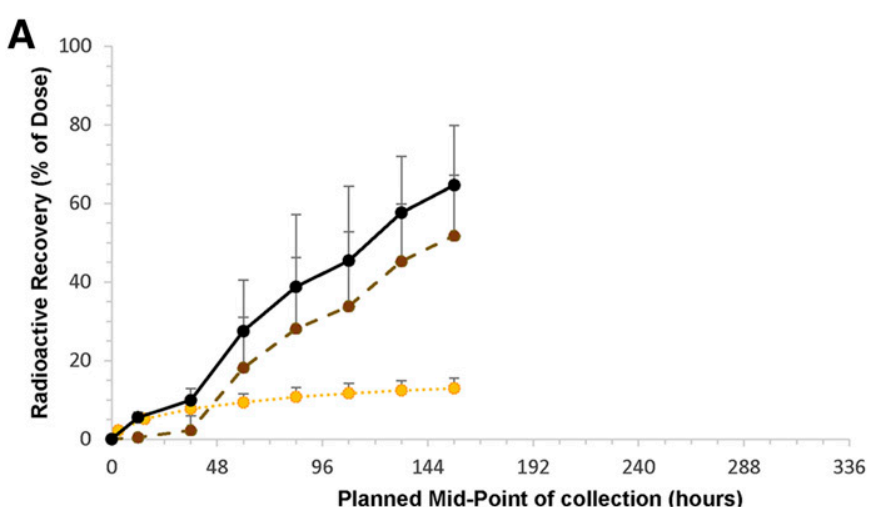

B

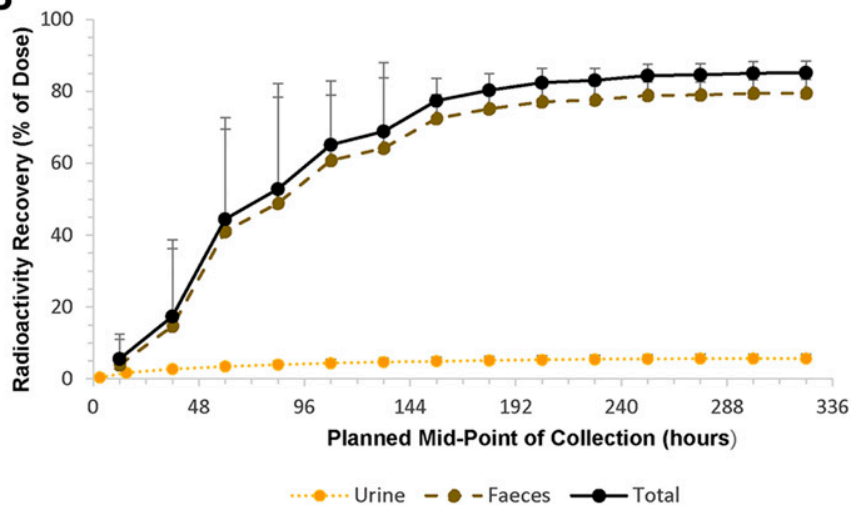

Fig. 2. Cumulative arithmetic mean (+S.D.) of radioactivity recovery over time following intravenous (A) and oral (B) administration of $\left[{ }^{14} \mathrm{C}\right]$ nemiralisib.

plates were sealed prior to scintillation counting. Each well was counted using a microplate scintillation counter (Packard Topcount NXT; Perkin Elmer) with 12 independent photomultiplier tubes and no background subtraction. The scintillation data were converted into a form compatible with Laura software using its "LSC import" function to reconstruct radio-chromatograms. Off-line radiodetection by AMS was conducted based on a previously reported method used to characterize inhaled vilanterol (Harrell et al., 2013), except in the current study UPLC was deployed with a flow rate of $0.3 \mathrm{ml} / \mathrm{min}$ compared with a flow rate of $1 \mathrm{ml} / \mathrm{min}$ using conventional HPLC for vilanterol. Fractions were collected from the column eluate post sample injection using a CTC Analytics HTX Pal system. The timing for collection was pre-set depending on sample acquired. Additional details are provided in the Supplemental Methods and in published literature (Young et al., 2014). Metabolite structures were assigned by comparison of relative retention times to pre-existing data from in vitro human hepatocyte preparations (unpublished GSK data).

\section{Safety}

Safety was assessed by measurement of vital signs, 12-lead ECGs and routine laboratory tests before dosing, over the first 24 hour post-dose and prior to discharge. Adverse events were monitored throughout the study.

\section{Pharmacokinetics and Statistical Analysis}

The sample size of 6 was deemed appropriate to investigate the primary objective (Penner et al., 2009). Plasma nemiralisib (parent drug following inhaled or oral administration), $\left[{ }^{14} \mathrm{C}\right]$ nemiralisib (parent drug following intravenous administration), and total radioactivity concentration-time data were analyzed by non-compartmental methods with WinNonlin Version 7.0, using the actual sampling times recorded during the study for derivation of PK parameters. Nemiralisib plasma kinetics in terms of clearance (CL), volume of distribution at steady-state, together with the bioavailability via each route (inhaled/oral) of administration were calculated.

Several parameters calculated in this publication did not form part of the original study plan and are, therefore, deemed calculated post hoc. Blood
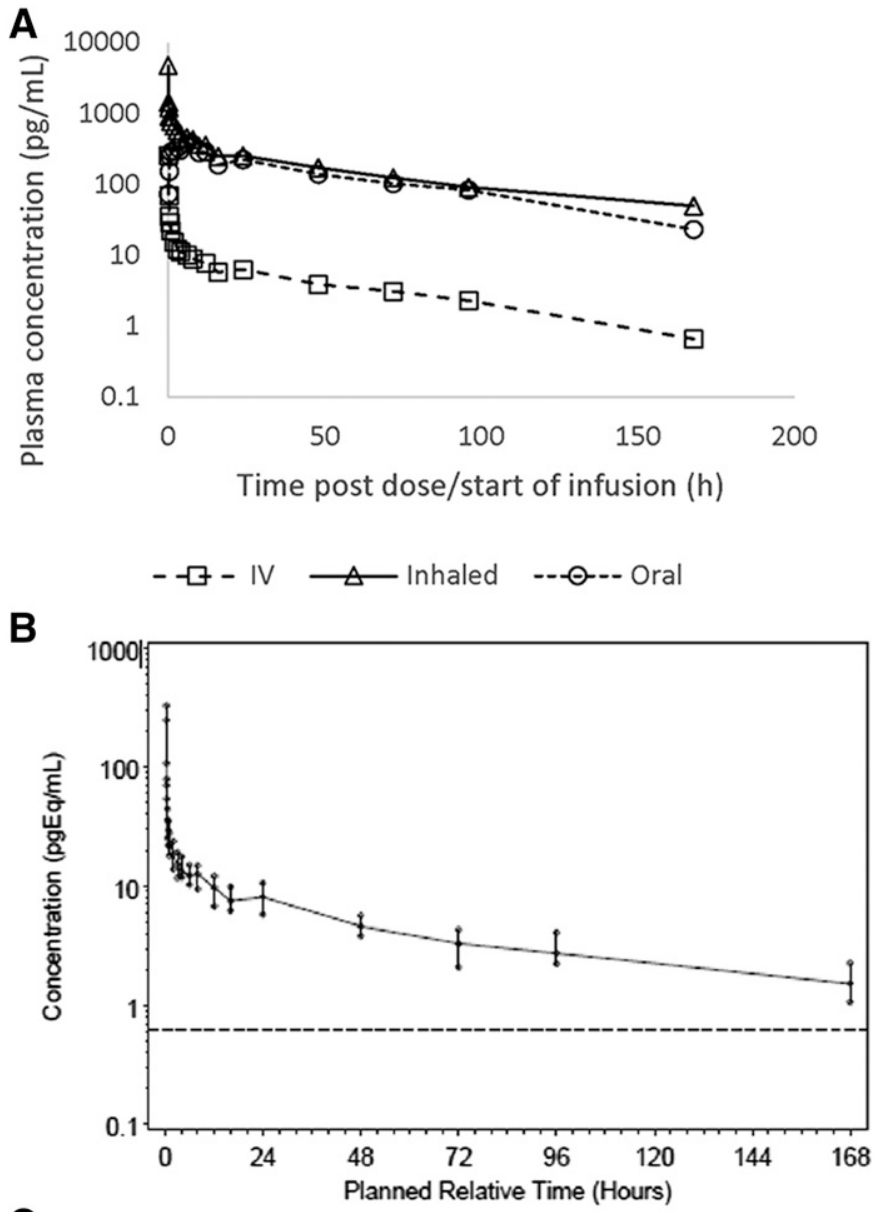

C

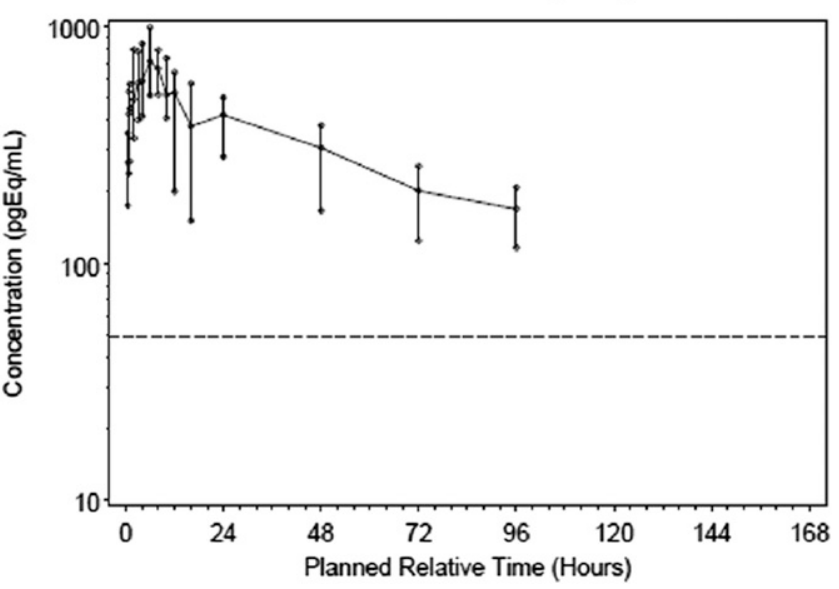

Fig. 3. Median (and range; $\mathrm{B}$ and $\mathrm{C}$ only) concentrations of (A) plasma nemiralisib following $10 \mu \mathrm{g}$ intravenous $\left[{ }^{14} \mathrm{C}\right]$ nemiralisib $+1000 \mu \mathrm{g}$ inhaled nemiralisib and $800 \mu \mathrm{g}$ oral $\left[{ }^{14} \mathrm{C}\right]$ nemiralisib; (B) total radioactivity in plasma following intravenous administration; and $(\mathrm{C})$ total radioactivity in plasma following oral administration.

clearance was calculated post hoc as plasma clearance/blood:plasma ratio where blood:plasma ratio was assumed to be of 1.08 (GSK unpublished data). Percentage liver blood flow was also calculated post hoc as blood clearance/ human hepatic blood flow, which was assumed to be $87 \mathrm{l} / \mathrm{h}$ (Davies and Morris, 1993). $F_{\text {lung }}$ was calculated post hoc with reference to nemiralisib data generated in other clinical study publications. Equations used to characterize various absorption and first-pass pharmacokinetic parameters are provided in Table 1, which include those calculated post hoc. This post hoc analysis included all derivations of first-pass and absorption parameters (with the exception of ML), as 
TABLE 3

Nemiralisib absolute bioavailability following inhaled delivery and oral dosing

All parameters calculated from individual data as presented in Supplemental Table 1.

\begin{tabular}{lllll}
\hline & & & \multicolumn{2}{c}{ Absolute Bioavailability (\%) } \\
\cline { 4 - 5 } $\begin{array}{l}\text { Test Route of } \\
\text { Administration }\end{array}$ & Parameter & $n$ & $\begin{array}{c}\text { Geometric Mean } \\
(\mathrm{CVb} \%)\end{array}$ & $\begin{array}{c}\text { 95\% Confidence } \\
\text { Intervals }\end{array}$ \\
\hline \multirow{2}{*}{ Inhaled } & $\mathrm{F}_{0-\text { inf }}$ & 4 & $38.07(19.7)$ & $(27.93,51.89)$ \\
& $\mathrm{F}_{0-t}$ & 6 & $42.13(21.8)$ & $(33.59,52.84)$ \\
Oral & $\mathrm{F}_{0-\text { inf }}$ & 4 & $35.15(3.35)$ & $(33.32,37.07)$ \\
& $\mathrm{F}_{0-t}$ & 6 & $32.61(12.8)$ & $(28.54,37.28)$ \\
\hline
\end{tabular}

detailed in Table 4. Three different approaches were used to characterize $\mathrm{F}_{\mathrm{abs}}$. In eqs. 1 and $2, \mathrm{~F}_{\mathrm{abs}}$ is calculated by comparing total drug-related material in urine (eq. 1) or plasma (eq. 2) between intravenous and oral doses. A third approach uses other first-pass parameters (eq. 9) and has been used to calculate $F_{\text {abs }}$ with and without a consideration of renal clearance of parent nemiralisib $\left(\mathrm{CL}_{\mathrm{r}}\right) . \mathrm{CL}_{\mathrm{r}}$ was calculated using eqs. 3 and 4 from the amount of unchanged nemiralisib (amount excreted (Ae) urine - post hoc) derived from the metabolite profile collected following oral administration in preference to intravenous administration where radioactivity concentrations were substantially lower. A maximum renal clearance of total radioactivity (total drug-related material) in urine is provided for the intravenous dose in Table 2 and has not been used for any further mathematical derivations. Further equations are provided in Table 1 and the corresponding notes to characterize $\mathrm{E}_{\mathrm{h}}$ (eq. 5), $\mathrm{F}_{\mathrm{h}}$ (eq. 6), and $\mathrm{F}_{\mathrm{g}}$ (eqs. 7 and 8) were all calculated post hoc. Since a value for nemiralisib renal clearance was not available, $\mathrm{CL}_{\mathrm{r}}$ in eq. 5 (post hoc from urine metabolite profile) was initially set to zero and $\mathrm{F}_{\mathrm{h}}$ in eq. 8 was set to 1 (using a worst-case hepatic load of $100 \%$ ). $\mathrm{E}_{\mathrm{h}}$ (eq. 5), $\mathrm{F}_{\mathrm{h}}$ (eq. 6), and $\mathrm{F}_{\mathrm{g}}$ (eqs. 7 and 8) were recalculated, correcting for $\mathrm{CLr}$, for comparison of these values when calculated with and without a correction for $\mathrm{CL}_{\mathrm{r}}$.

\section{Results}

Demographic and Baseline Characteristics. Seven men were screened, of whom six were enrolled and completed the study. Their mean (S.D.) age was 43 (10) years with a mean (S.D.) body mass index of $28(3.8) \mathrm{kg} / \mathrm{m}^{2}$.

Mass Balance and Excretion. The mean recovery of radioactivity over time following intravenous and oral administration of $\left[{ }^{14} \mathrm{C}\right]$ nemiralisib is presented graphically in Fig. 2. Excretion of $\left[{ }^{14} \mathrm{C}\right]$

\section{TABLE 5}

Quantification of the major radioactive components in plasma extracts following intravenous or oral administration of $\left[{ }^{14} \mathrm{C}\right]$ nemiralisib

\begin{tabular}{|c|c|c|}
\hline \multirow[b]{3}{*}{ ID } & \multirow{2}{*}{\multicolumn{2}{|c|}{$\begin{array}{c}\begin{array}{c}\% \text { Sample Radioactivity (pg } \\
\text { equivalents }\left[{ }^{14} \mathrm{C}\right] \text { nemiralisib/g) }\end{array} \\
\text { Plasma }\end{array}$}} \\
\hline & & \\
\hline & $\begin{array}{c}\text { Intravenous } \\
0-24 \mathrm{~h}\end{array}$ & $\begin{array}{c}\text { Oral } \\
0-24 \mathrm{~h}\end{array}$ \\
\hline $\mathrm{P}$ (nemiralisib) ${ }^{a}$ & $87.6(47.3)$ & $76.3(511)$ \\
\hline M1(GSK2330979) and/or M9 & $8.4(4.6)$ & $10.0(66.7)$ \\
\hline $\begin{array}{l}\text { Total radioactive material assigned } \% \\
\text { (pg equivalents }\left[{ }^{14} \mathrm{C}\right] \text { nemiralisib/g) }\end{array}$ & $96.0(51.9)$ & $86.3(578)$ \\
\hline Total pg equivalents $\left[{ }^{14} \mathrm{C}\right]$ nemiralisib/g in pool & 54.0 & 670 \\
\hline
\end{tabular}

${ }^{a}$ Identified by LC-MS, all other peaks identified by comparison of relative retention times to pre-existing data from in vitro human hepatocytes.

nemiralisib-related material was slow and protracted irrespective of the route of administration. Following oral administration of $\left[{ }^{14} \mathrm{C}\right]$ nemiralisib, a total of $85 \%$ of the administered dose was recovered by 14 days after dosing with $79 \%$ being recovered in feces and $6 \%$ in the urine. Following intravenous administration of $\left[{ }^{14} \mathrm{C}\right]$ nemiralisib (in combination with an inhaled non-radiolabeled dose), $65 \%$ of the administered dose was recovered by 7 days after dosing with $52 \%$ recovered in feces (approximately $80 \%$ of the total recovered radioactivity) and $13 \%$ in the urine (approximately $20 \%$ of the total recovered radioactivity).

Pharmacokinetic Results. Following inhaled administration, maximum nemiralisib plasma concentrations $\left(C_{\max }\right)$ were attained rapidly (median $t_{\text {max }}$ : 0.033 hours; 2 minutes) with a steep decline in concentrations immediately after dosing, followed by a slower decline and a geometric mean terminal phase half-life $\left(t_{1 / 2}\right)$ of 58.4 hours (Fig. 3A; Table 2). The geometric mean absolute bioavailability following inhaled nemiralisib was $38 \%$ (95\% CI: $28 \%, 52 \%$ ) based on area under the plasma concentration time curve from time zero extrapolated to infinite time (Table 3). Small fluctuations (secondary peaking) in plasma concentrations were observed over the first 24 hour post dose. At the end of intravenous infusion, for both total radioactivity and nemiralisib, median $t_{\max }$ was 0.233 hour (14 minutes) with geometric mean $C_{\max }(204 \mathrm{pg} \mathrm{Eq} / \mathrm{ml}$ and $206 \mathrm{pg} / \mathrm{ml}$, respectively) being

TABLE 4

Summary of metabolic, first-pass, and absorption pharmacokinetic parameters

Parameters calculated from individual data as presented in Supplemental Table 1. Blood:plasma ratio for nemiralisib is assumed to be 1.08 (measured unpublished value). Blood:plasma ratio for nemiralisib drug-related material (radioactivity) was not measured and is assumed to be $1 . \mathrm{CL}_{\mathrm{r}}$ was calculated from urine metabolic profile and assumed to be $0.93 \mathrm{l} / \mathrm{h}$ (Table 2 ).

\begin{tabular}{|c|c|c|c|c|c|c|}
\hline Parameter & Treatment & Equation & $N$ & $n$ & Geometric Mean $(\mathrm{CVb} \%)$ & 95\% Confidence Intervals \\
\hline $\operatorname{ML}(\text { i.v. })^{a, b}$ & i.v. & eq. 7 & 6 & 6 & $0.252(0.203)$ & $(0.039,0.465)$ \\
\hline $\operatorname{ML}(\text { oral })^{a}$ & Oral & eq. 7 & 6 & 6 & $0.435(33.8)$ & $(0.308,0.614)$ \\
\hline \multicolumn{7}{|c|}{ First pass pharmacokinetic parameters calculated with correction for $\mathrm{CLr}^{c}$} \\
\hline $\mathrm{E}_{\mathrm{h}}$ (blood) & i.v. & eq. 5 & 6 & 4 & $0.103(51.2)$ & $(0.048,0.223)$ \\
\hline $\mathrm{F}_{\mathrm{h}}$ (blood) & Oral & eq. 6 & 6 & 4 & $0.884(7.55)$ & $(0.784,0.997)$ \\
\hline $\mathrm{F}_{\mathrm{g}}$ (blood) & Oral & eq. 8 & 6 & 4 & $0.829(27.3)$ & $(0.541,1.27)$ \\
\hline $\mathrm{F}_{\mathrm{abs}}($ blood $)$ & Oral & eq. 9 & 6 & 4 & $0.480(25.1)$ & $(0.324,0.711)$ \\
\hline \multicolumn{7}{|c|}{ First pass pharmacokinetic parameters calculated assuming CLr is negligible ${ }^{d}$} \\
\hline $\mathrm{E}_{\mathrm{h}}$ (blood) & i.v. & eq. 5 & 6 & 4 & $0.115(46.7)$ & $(0.057,0.233)$ \\
\hline $\mathrm{F}_{\mathrm{h}}(\text { blood })^{c}$ & Oral & eq. 6 & 6 & 4 & $0.873(7.64)$ & $(0.774,0.986)$ \\
\hline $\mathrm{F}_{\mathrm{g}}(\text { blood })^{e}$ & Oral & eq. 8 & 6 & 6 & $0.785(21.3)$ & $(0.630,0.979)$ \\
\hline $\mathrm{F}_{\mathrm{abs}}($ blood $)$ & Oral & eq. 9 & 6 & 4 & $0.549(23.8)$ & $(0.378,0.798)$ \\
\hline \multicolumn{7}{|c|}{ Other methods to calculate $\mathrm{F}_{\mathrm{abs}}{ }^{e}$} \\
\hline $\mathrm{F}_{\mathrm{abs}}$ & Oral & eq. 1 & \multicolumn{2}{|c|}{ Pooled Mean } & 0.450 & - \\
\hline $\mathrm{F}_{\mathrm{abs}}$ & Oral & eq. 2 & 6 & 6 & $0.441(25.9)$ & $(0.337,0.576)$ \\
\hline
\end{tabular}

$N$, number of subjects; $n$, number of evaluable subjects.

${ }^{a}$ When $\mathrm{AUC}_{0 \text {-inf }}$ values were unavailable for calculating metabolite load (ML), AUC $\mathrm{A}_{0-t}$ was used. Specifically, AUC $\mathrm{C}_{0 \text {-inf }}$ was only used for subject 5 (both routes) and subject 1 (i.v. only) - see Supplemental Table 1 .

${ }^{b}$ ML (i.v.) is presented as an arithmetic mean, S.D., and corresponding 95\% CI since geometric mean could not be calculated due to one negative value

${ }^{c}$ Parameters have been corrected for renal clearance $\left(\mathrm{CL}_{\mathrm{r}}\right)$ once parameter was available from urinary metabolism work.

${ }^{d}$ Parameters calculated prior to the availability of CLr. CLr is assumed "zero" for values calculated from eqs. 5, 6, and 9. Fh is assumed to be "1" for the calculation of $\mathrm{F}_{\text {abs }}$ using eq. 9 .

${ }^{e} \mathrm{~F}_{\mathrm{g} \text { blood }}=\mathrm{F}_{\mathrm{g} \text { plasma }}$ when blood:plasma ratio of drug-related material and $\mathrm{F}_{\mathrm{h}}$ are assumed to be 1 . 


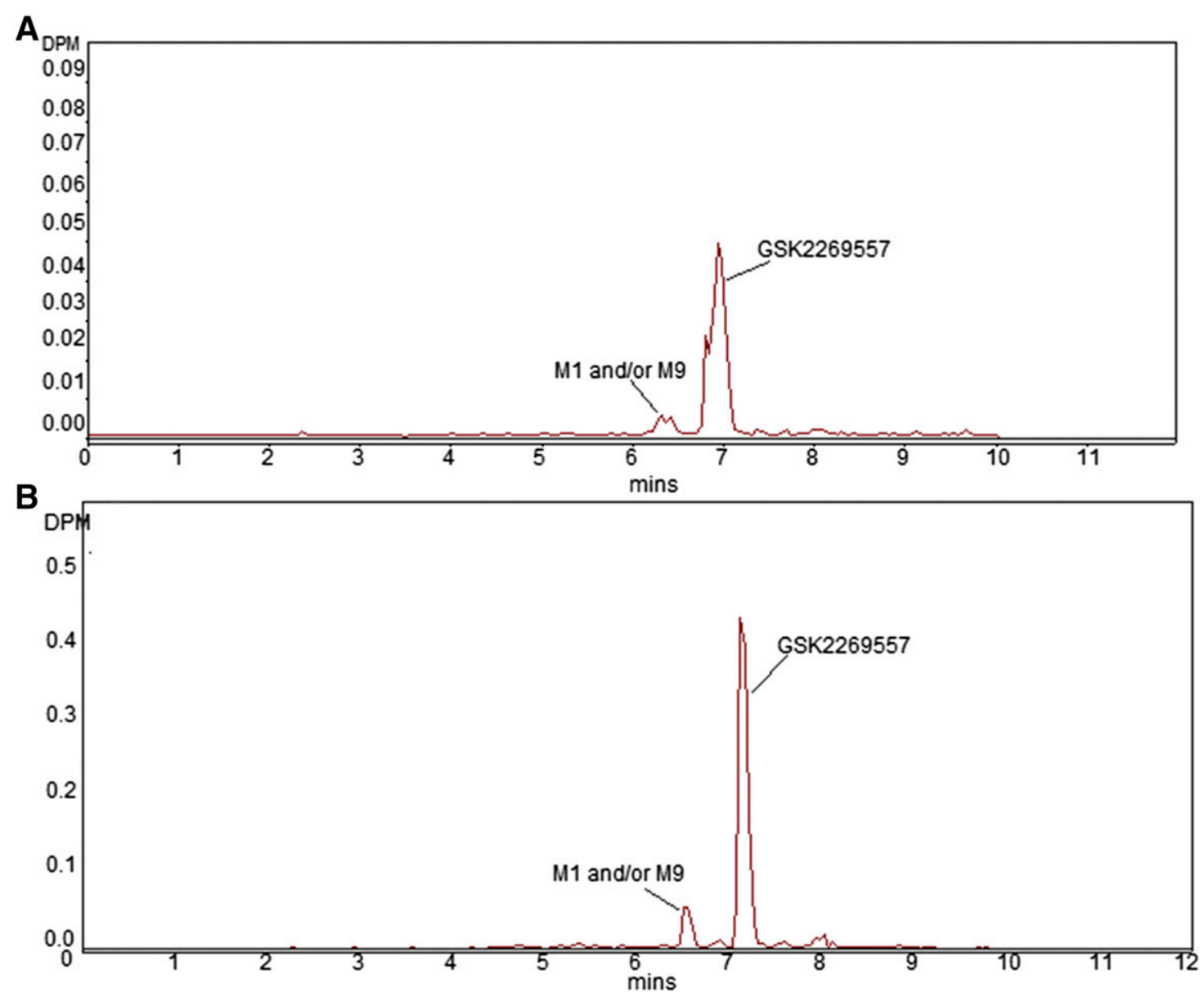

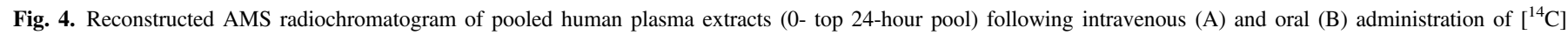
nemiralisib. DPM, disintegrations/min.

followed by an initial rapid then slower decline in concentrations (Fig. 3; Table 1). A small increase in nemiralisib plasma concentration (secondary peak) was observed at 24 hours. intravenous plasma nemiralisib concentrations rapidly declined from $C_{\max }$ to $32.7 \mathrm{pg} / \mathrm{ml}$ at 0.5 hour post dose with a $t_{1 / 2}$ of 54.7 hours. The geometric mean clearance of nemiralisib from plasma was low $(10.8 \mathrm{l} / \mathrm{h})$ and geometric mean volume of distribution at steady state was high (728 1). By correcting plasma clearance with a human blood:plasma ratio of 1.08 (unpublished GSK data), the corresponding human blood clearance is $10.0 \mathrm{l} / \mathrm{h}$ $(10.8 / 1.08)$ or $11 \%$ liver blood flow compared with a human hepatic blood flow of 87 1/h (Davies and Morris, 1993).

Following oral administration, nemiralisib plasma profiles indicated a protracted absorption phase with a median time to peak levels of 6 hour post dose followed by a slow decline in drug levels. Geometric mean $t_{1 / 2}$ following oral dosing was 50.8 hours, within a similar range to that observed with intravenous dosing (Fig. 3; Table 1). Geometric mean absolute bioavailability of $\left[{ }^{14} \mathrm{C}\right]$ nemiralisib following oral administration of an $800 \mu \mathrm{g}$ dose (mean radiometric dose of $769 \mu \mathrm{g}$ ), was $35 \%(95 \%$ CI: $33 \%, 37 \%)$ based on area under the plasma concentration time curve from time zero extrapolated to infinite time (Table 2). Similar to the inhaled nemiralisib profile, small fluctuations in nemiralisib plasma concentrations (secondary peaking) were observed following both intravenous and oral administration. The geometric mean area under the plasma concentration time curve from time zero to last time of quantifiable concentration ratio indicated that nemiralisib accounted for $71 \%$ and $53 \%$ of total circulating radioactivity in the plasma following intravenous and oral administration, respectively.
Absorption and First-pass Pharmacokinetic Parameters. The absorption parameters of nemiralisib are described in Table 4 with geometric means being presented in the text. A value of 0.549 was originally derived for $\mathrm{F}_{a b s}$ using eq. 9 and assuming a negligible $\mathrm{CL}_{\mathrm{r}}$ since parent nemiralisib $\mathrm{CL}_{\mathrm{r}}$ was not available at the time of reporting. Herein we have compared this value (0.549) to several other methods that can be used to estimate $F_{a b s}$, including the equivalent eq. 9 but incorporating an adjustment for $\mathrm{CL}_{\mathrm{r}}$. Lower estimates of $\mathrm{F}_{\mathrm{abs}}$ are derived when comparing urinary excretion $(0.450$; eq. 1$)$ or plasma radioactivity AUC (0.441; eq. 2$)$ between oral and intravenous administrations. When the uncorrected value $(0.549)$ is corrected for renal blood clearance using eq. 9 the revised estimate $(0.480)$ is closer to those following application of eqs. 1 and 2. This revised estimate (0.480) has been used for the purposes of this publication as the true estimate of $F_{a b s}$. There were small changes to $F_{h}, F_{\text {oral }}$, and $F_{g}$ depending on whether a correction for $\mathrm{CL}_{\mathrm{r}}$ was applied or not; again, all corrections using $\mathrm{CL}_{\mathrm{r}}$ have been applied for the purposes of this publication after the original study was reported.

Inhaled Disposition. An estimate of $\mathrm{F}_{\text {lung }}$ (the inhaled dose absorbed through the lung) can be calculated by integrating the data reported for this clinical study with charcoal block data from a previously published nemiralisib clinical study (Wilson et al., 2018). The fraction of drug orally absorbed following inhaled delivery represents $23 \%$ of the resulting systemic exposure (Wilson et al., 2018), which, when compared with $F_{\text {inh }}(38 \%)$, means that $(0.23 \% \times 38 \%) 8.7 \%$ of the inhaled exposure is coming from the oral absorption making the inhaled fraction via the lung $\left(\mathrm{F}_{\text {lung }}\right)$ 

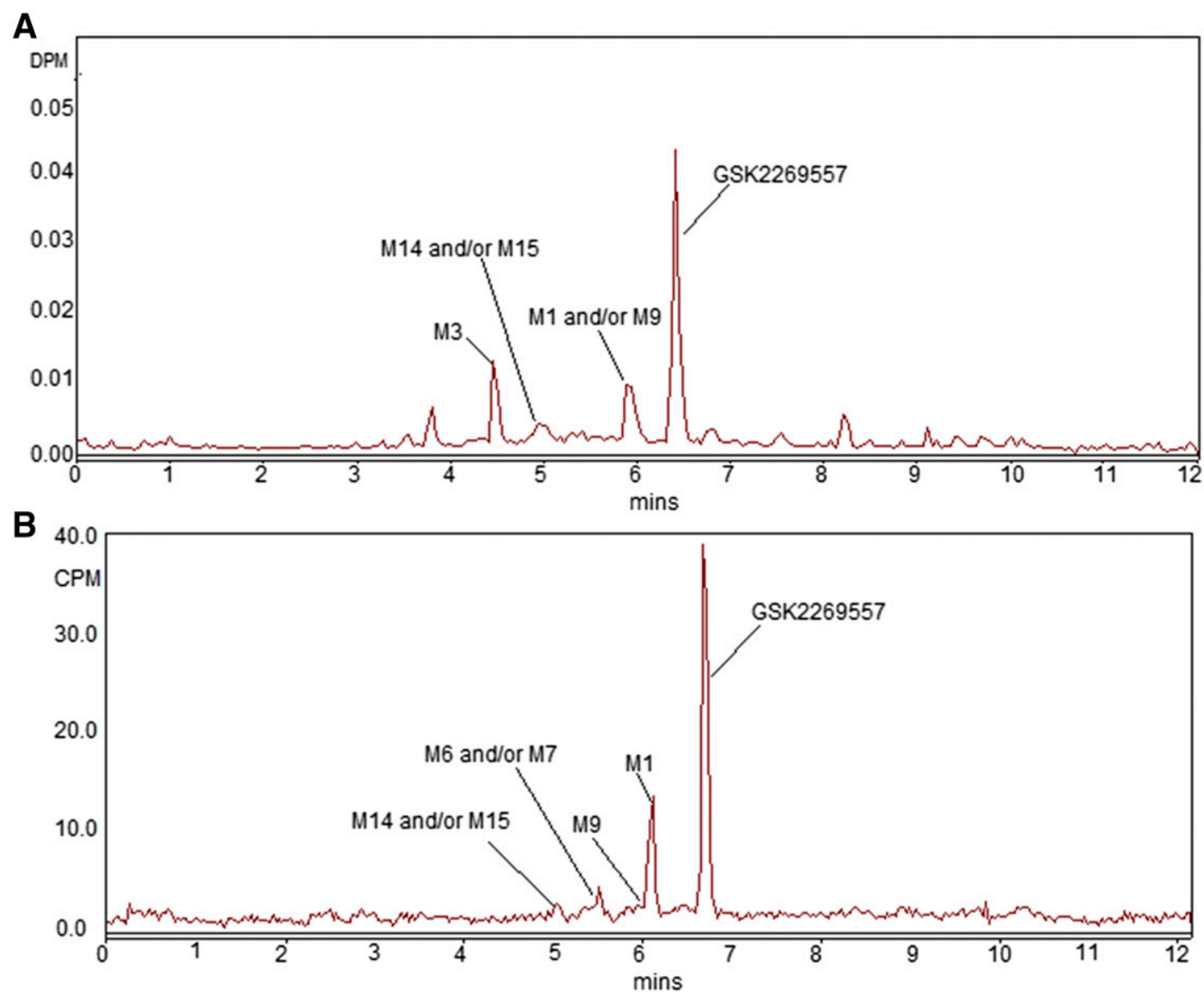

Fig. 5. (A) Reconstructed AMS radiochromatogram of pooled human urine and (B) Reconstructed TopCount radiochromatogram of pooled human feces, following oral administration of $\left[{ }^{14} \mathrm{C}\right]$ nemiralisib. CPM, counts/min; DPM, disintegrations/min .

$(38 \%-8.7 \%)=29 \%$. These calculations do not form part of the clinical study reporting but demonstrate the utility of integrating data across different studies.

Metabolism. AMS analysis of time resolved pooled plasma (0-24 hours) extracts from intravenous and oral dosed subjects showed that nemiralisib was the major circulating component $(88 \%$ and $76 \%$ of plasma radioactivity, 47.3 and $511 \mathrm{pgEq} / \mathrm{ml}$, respectively) (Table 5). The other notable radiolabeled component $(\sim 8 \%$ and $10 \%$ of the samples radioactivity, 4.55 and $66.7 \mathrm{pgEq} / \mathrm{ml}$, respectively) was identified as metabolite M1 (GSK2330979, formed by $N$-dealkylation) and/or M9

TABLE 6

Quantification of the major radioactive components in urine, feces and bile extracts following intravenous or oral administration of $\left[{ }^{14} \mathrm{C}\right]$ nemiralisib

\begin{tabular}{|c|c|c|c|c|}
\hline \multirow{3}{*}{ ID } & \multicolumn{4}{|c|}{ Mean \% Matrix Radioactivity (Mean \% Dose) } \\
\hline & \multirow{2}{*}{$\begin{array}{c}\text { Urine } \\
\text { Oral } \\
\text { 6-Subject pool }\end{array}$} & \multicolumn{2}{|c|}{ Feces } & \multirow{2}{*}{$\begin{array}{c}\text { Bile } \\
\text { Intravenous } \\
\text { 6-Subject poo }\end{array}$} \\
\hline & & $\begin{array}{c}\text { Intravenous } \\
\text { 6-Subject pool }\end{array}$ & $\begin{array}{c}\text { Oral } \\
\text { 6-Subject pool }\end{array}$ & \\
\hline $\mathrm{P}$ (nemiralisib) & $43.3(2.2)$ & $13.2(6.8)$ & $41.0(21.6)$ & 52.4 \\
\hline M3 & $11.9(0.6)$ & ND & ND & ND \\
\hline M1(GSK2330979) and/or M9 & $14.7(0.8)$ & $8.3(4.3)$ & $14.7(7.8)^{a} 1.8(1.0)^{b}$ & 12.1 \\
\hline M6 and/or M7 & ND & $8.8(4.6)$ & $4.4(2.3)$ & 6.3 \\
\hline M14 and/or M15 & $7.6(0.4)$ & $12.8(6.6)$ & $2.0(1.1)$ & 7.4 \\
\hline Total radioactive material assigned ${ }^{c}$ & $77.4(4.0)$ & $43.1(22.3)$ & $63.9(33.7)$ & 78.1 \\
\hline$\%$ Administered dose in matrix pool ${ }^{d}$ & 5.2 & 51.7 & 72.1 & N/A \\
\hline Total $\%$ dose excreted in matrix ${ }^{e}$ & 5.66 & 51.7 & 79.5 & N/A \\
\hline
\end{tabular}

${ }^{a} \mathrm{M} 1$ (GSK2330979)

${ }^{b} \mathrm{M} 9$

${ }^{c}$ Total radioactive material assigned is the sum of the $\%$ matrix radioactivity (and \% dose) for all identified metabolites in the analyzed pool.

${ }^{d} \%$ Administered dose in the matrix pool represents approximately $\geq 90 \%$ of the radioactivity excreted via that route and is quoted for all matrices other than bile Bile string collection is not quantitative.

${ }^{e}$ Total \% dose excreted in matrix is the mean figure from the ADME study. 

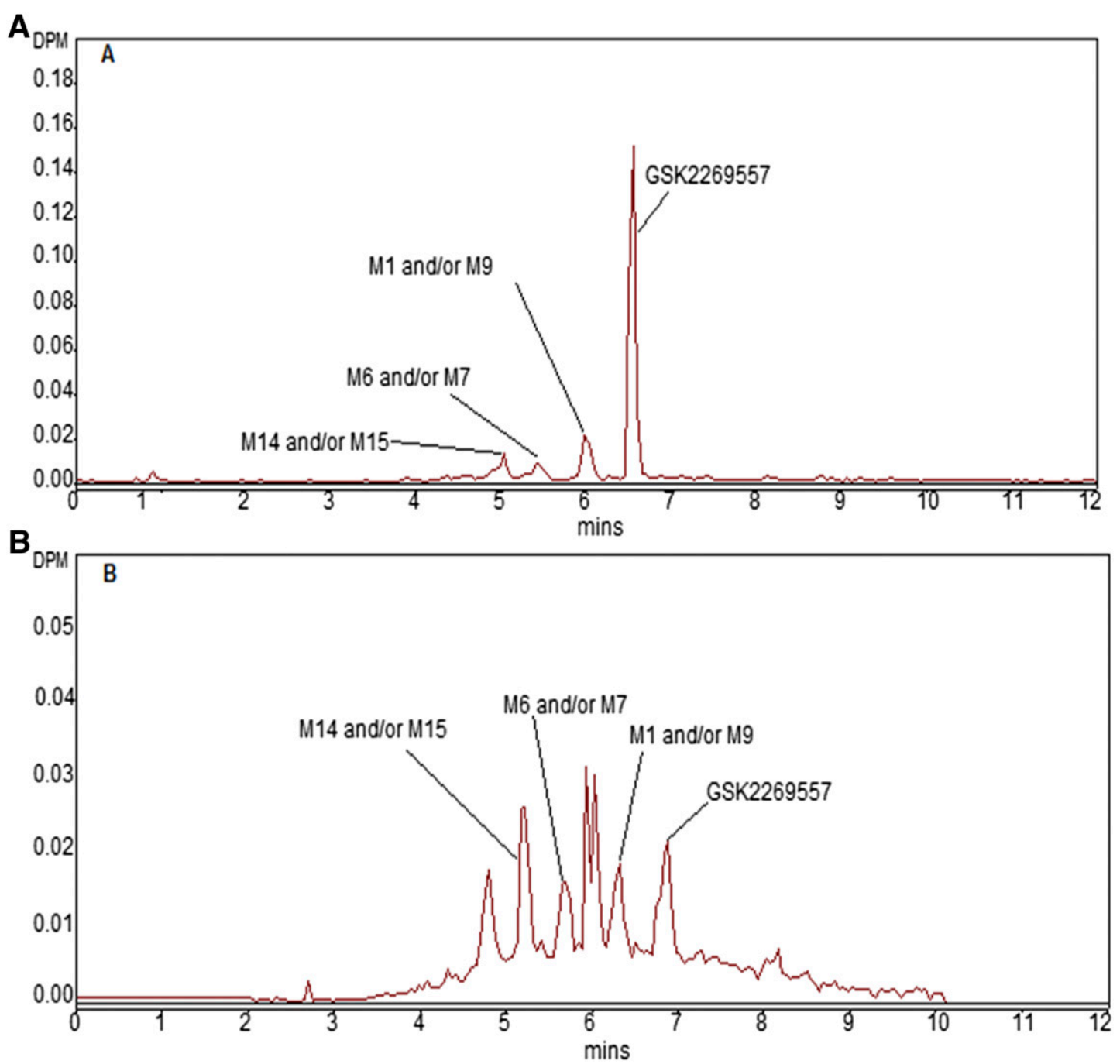

Fig. 6. Reconstructed AMS radiochromatogram of pooled human duodenal bile extract (A) and pooled human feces extract (B), following intravenous administration of $\left[{ }^{14} \mathrm{C}\right]$ nemiralisib. DPM, disintegrations/min.

(formed by $N$-dealkylation and sulfate conjugation), which had both shown comparable relative retention times in human hepatocytes to that observed for the plasma component here. Representative AMS chromatograms of reconstituted pooled plasma extracts are shown in Fig. 4.

Following oral dosing, unchanged nemiralisib was the principal radioactive component in pooled urine extracts $(43 \%$ of the AMS radiochromatogram, $\sim 2 \%$ of the administered dose) and feces ( $41 \%$ of the radiochromatogram or $\sim 22 \%$ of the dose) (Fig. 5; Table 6). By applying eqs. 3 and 4 (Table 1), this corresponded to a low renal blood clearance of unchanged nemiralisib of $0.93 \mathrm{l} / \mathrm{h}$, which was in line with the overestimated renal clearance approximated from total radioactivity excreted in urine from the intravenous dose $(1.25 \mathrm{l} / \mathrm{h})$. Other notable radioactive peaks on the urine AMS radiochromatogram $(\sim 12 \%, 15 \%$, and $8 \%$ of the sample radioactivity; $0.6 \%, 0.8 \%$, and $0.4 \%$ of the dose, respectively) were identified as M3 (formed by oxidation and glucuronide conjugation), M1 and/or M9, and M14 (formed by direct glucuronide conjugation) and/or M15 (formed by oxidation). In feces, the $N$-dealkylated metabolite (M1, GSK2330979) was another major component ( $\sim 15 \%$ of the chromatogram or $8 \%$ of the dose), while other minor metabolites represented $\leq 4 \%$ of the chromatogram (M9, M6, and/or M7; M14 and/or M15) (Fig. 5; Table 6).

Following intravenous dosing, unchanged nemiralisib was also the main component seen in duodenal bile extract $(52 \%$ of the chromatogram) with other minor metabolites (expressed as \% of the chromatogram) identified as M1 and/or M9 (12\%), M6 and/or M7 (6\%), and M14 and/or M15 (7\%) (Fig. 6; Table 6). Unchanged nemiralisib was a lesser component in feces extracts following intravenous administration ( $13 \%$ of the chromatogram, or $\sim 7 \%$ of the administered dose) with other components (expressed as \% of the chromatogram) identified as M1 and/or M9 (8\%), M6 and/or M7 (9\%), and M14 and/or M15 (13\%) (Fig. 6; Table 6). A proposed metabolic scheme for nemiralisib is shown in Fig. 7.

Safety. No deaths, serious adverse events, or other notable adverse events were reported during the study, and no event led to withdrawal of the subject from the study or permanent discontinuation of the study treatment. Adverse events of headache (two episodes) and malaise (one episode) occurred in one of the six subjects in the study. Two subjects presented with neutrophil counts below $1.5 \times 10^{9} / 1$ not considered as adverse events or regarded as clinically significant. There were no clinically significant changes in vital signs, ECG, or urinalysis findings.

\section{Discussion}

An innovative clinical pharmacology trial design has been used to fully characterize human ADME and PK of inhaled nemiralisib in healthy men through integration of data derived from a microtracer 


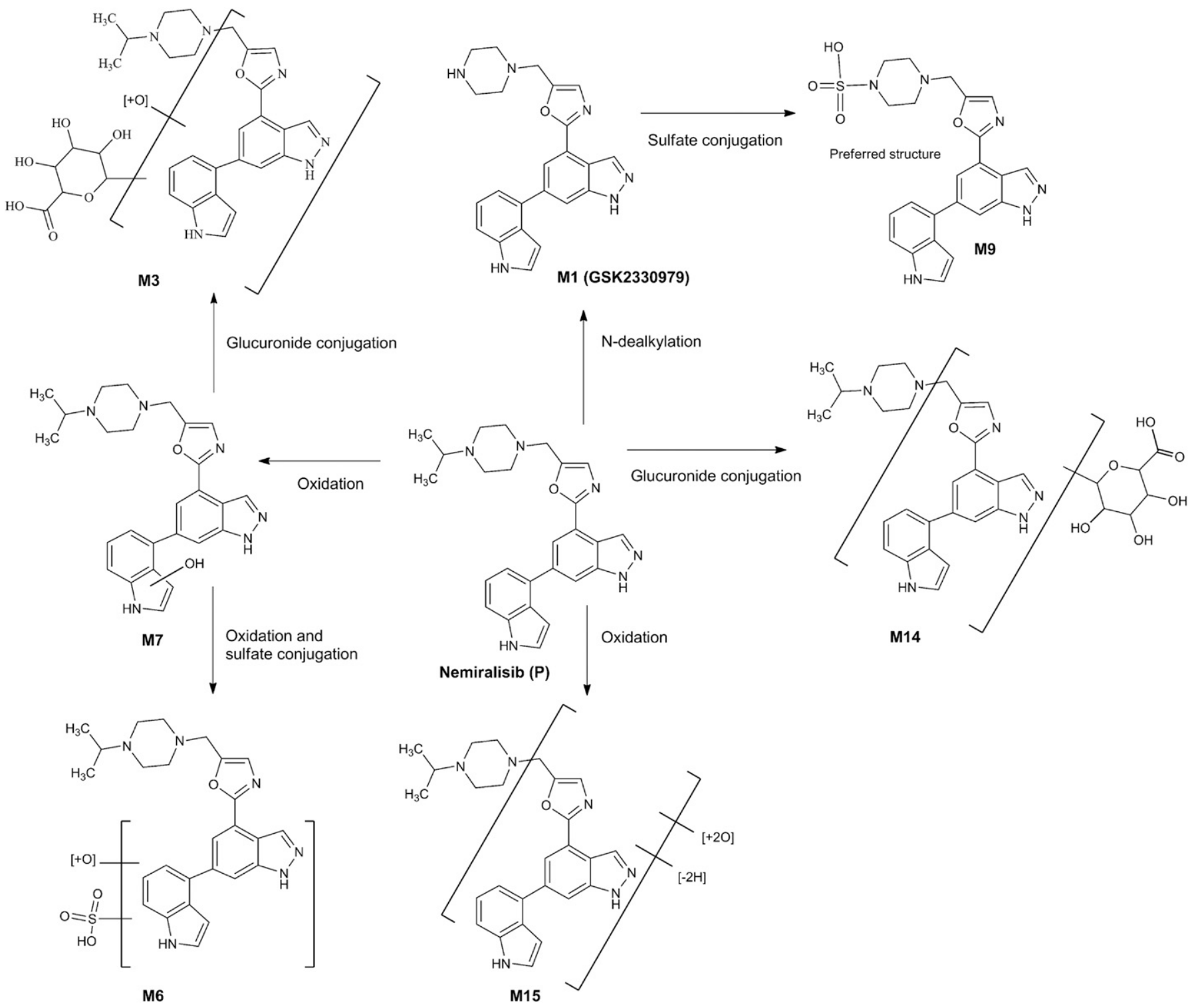

Fig. 7. Putative metabolic scheme for nemiralisib in human.

\begin{abstract}
${ }^{14} \mathrm{C}$ intravenous administration delivered concomitantly with an inhaled therapeutically relevant dose, followed by an oral ${ }^{14} \mathrm{C}$ administration after a washout of 14 days. The study used synergistic state-of-the-art analytical methods including AMS, which was configured to deliver highly sensitive nemiralisib specific plasma concentration levels, total drug related material concentrations in plasma, excretion balance data, and metabolite profiles in plasma, bile, and feces. The small two-part study has enabled the generation of intravenous, oral, and inhaled PK parameters including parameters such as $\mathrm{F}_{\mathrm{abs}}, \mathrm{F}_{\mathrm{g}}, \mathrm{E}_{\mathrm{h}}, \mathrm{F}_{\text {lung }}$, and $\mathrm{CL}_{\mathrm{r}}$, parameters that are not easily derived using conventional clinical $\mathrm{PK}$ designs. The incorporation of a ${ }^{14} \mathrm{C}$-isotope into the parent nemiralisib molecule enabled a full evaluation of nemiralisib metabolism in both plasma (to assess human metabolite exposure) and in excreta (to assess elimination pathways), with intravenous and oral administrations of $\left[{ }^{14} \mathrm{C}\right]$ nemiralisib being used as surrogates for the lungdeposited and swallowed portions of an inhaled dose, respectively. Pharmacological relevance of the intravenous microtracer dose was achieved through concomitant administration with a therapeutically relevant inhalation dose. Furthermore, metabolite profiling of human duodenal bile using the Entero-Test device enabled metabolites
\end{abstract}

eliminated through biliary elimination to be characterized, giving a more thorough understanding of elimination pathways, including enterohepatic recirculation. The microtracer study design published for inhaled batefenterol (Ambery et al., 2018) has been developed further to include the calculation of additional parameters and a more comprehensive metabolism assessment and is, to our knowledge, the first of its kind.

Consistent with its long terminal PK half-life, nemiralisib demonstrated a slow and protracted elimination of radioactivity. A retrospective analysis of total recoveries published for mass balance radioactive clinical studies concludes that acceptable target recoveries should be $>80 \%$ of the administered radioactive dose (Roffey et al., 2007). The total recovery of radioactivity achieved following oral administration of $1850 \mathrm{kBq}$ radioactivity in this study was $85 \%$, which is, therefore, acceptable. Although all subjects met the predetermined stopping criteria by day $15(<1 \%$ dose recovered in a 24-hour collection interval), the slow protracted elimination meant that further sample collections may well have improved this total recovery further. The lower radioactive dose $(22.2 \mathrm{kBq})$ delivered by intravenous administration, for logistical reasons 


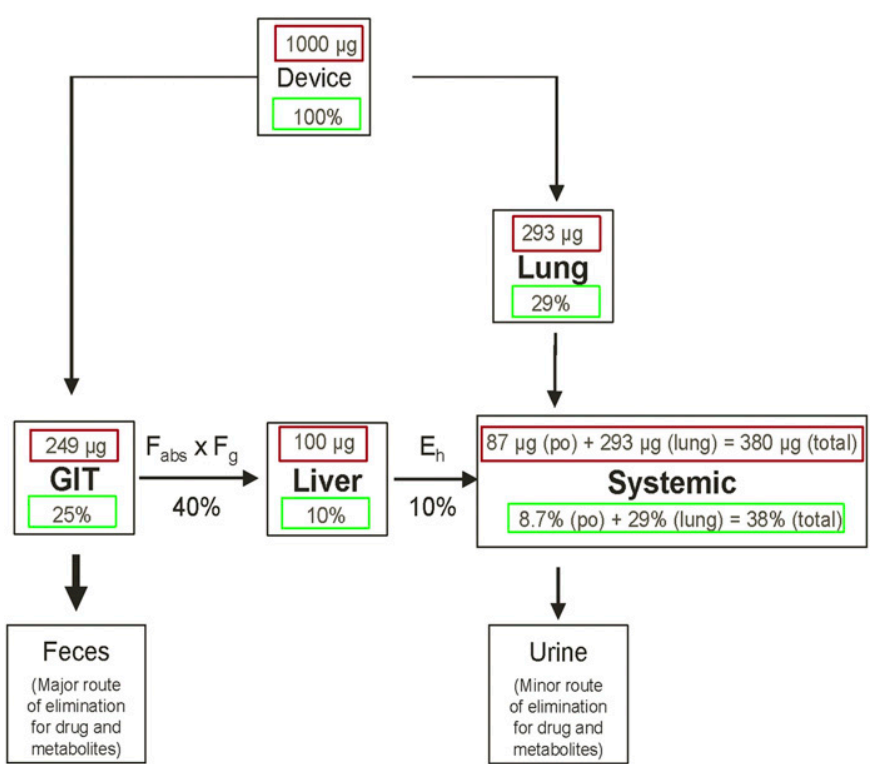

Fig. 8. Disposition scheme for inhaled nemiralisib based on the pharmacokinetic parameters derived following intravenous, oral (po), and inhalation dosing of nemiralisib to healthy men. The disposition scheme was constructed by first assuming that if $\mathrm{F}_{\mathrm{inh}}=0.38$, then $38 \%(380 \mu \mathrm{g})$ reaches the systemic compartment from an inhaled dose of $1000 \mu \mathrm{g}$. If $23 \%$ of the systemic exposure is due to a swallowed component ( $\mathrm{F}_{\text {swallowed; }}$ Wilson et al., 2018) then $9 \%(380 \mu \mathrm{g} * 23 \%=87 \mu \mathrm{g})$ of systemic drug is derived from that swallowed component. The difference then by subtraction is $29 \%(380-87 \mu \mathrm{g}=293 \mu \mathrm{g})$, which must reach the systemic compartment through the lung $\left(\mathrm{F}_{\text {lung }}\right)$. If $\mathrm{F}_{\text {oral }}$ is 0.35 , then $25 \%(87 \mu \mathrm{g} / 0.35=249 \mu \mathrm{g})$ must each reach the gastrointestinal tract (GIT) as swallowed drug. The systemic exposure derived from the oral component can also be estimated using absorption and first-pass parameters. By multiplying $\mathrm{F}_{\mathrm{abs}} * \mathrm{~F}_{\mathrm{g}}$; then $40 \%$ of swallowed nemiralisib escapes the GIT. Assuming 90\% escapes first-pass clearance $\left(1-E_{h}\right)$, then $8.9 \%(89 \mu \mathrm{g})$ reaches the systemic compartment from the swallowed dose, which compares well with $8.7 \%(87 \mu \mathrm{g})$ derived using $\mathrm{F}_{\text {inh }}$ and $\mathrm{F}_{\text {swallowed. With this approach, }}$ a total of $46 \%$ dose or $458 \mu \mathrm{g}(1000 \mu \mathrm{g}-293 \mu \mathrm{g}-249 \mu \mathrm{g})$ is unaccounted for, presumed remaining in device or exhaled.

precluded rapid data turnaround to allow recovery to be used as a release criterion, so a fixed duration collection of 7 days post dose was protocolled. Using AMS as a highly sensitive method to determine radioactive recovery, the total recovery from the intravenous administration was $65 \%$, which was approximately in line with the recovery over a similar period following oral administration.

Three of the approaches used to calculate $\mathrm{F}_{\mathrm{abs}}$ for nemiralisib in Table 4 were in close agreement $(0.450,0.441$, and 0.480 using eqs. 1,2 , and 9 correcting for $\mathrm{CL}_{\mathrm{r}}$ ). These three approaches provided a better estimate of $F_{a b s}$ than the uncorrected $F_{a b s}$ value of 0.549 , which was overestimated because it did not consider $\mathrm{CL}_{\mathrm{r}}$. This difference highlights the importance of correcting for $\mathrm{CL}_{\mathrm{r}}$ when using absorption and first-pass equations and is particularly important for molecules with higher $\mathrm{CL}_{\mathrm{r}}$. Nemiralisib was determined to be moderately well absorbed, regardless of the approach used to calculate $\mathrm{F}_{\mathrm{abs}}$.

Intravenous administration of $\left[{ }^{14} \mathrm{C}\right]$ nemiralisib resulted in plasma concentration/time profiles of unchanged nemiralisib characterized by multiexponential distribution and elimination. Nemiralisib was rapidly distributed followed by slower distribution profiles with volume of distribution at steady state (728 1) exceeding total body water (42 1; Davies and Morris, 1993), suggestive of extensive distribution of the drug beyond the plasma compartment. The drug exhibited relatively low blood clearance corresponding to $11 \%$ human liver blood flow. Oral administration resulted in a protracted absorption phase followed by a biexponential decline in drug concentrations. Any swallowed nemiralisib, including that from an inhaled dose, is likely to be relatively well absorbed across the gastrointestinal (GI) tract $\left(\mathrm{F}_{\mathrm{abs}}=0.44-0.48\right.$, Table 4; excluding values where CLr is assumed negligible) and then subject to poor first-pass extraction by the gut wall $\left(\mathrm{F}_{\mathrm{g}}=0.83\right)$ and liver $\left(E_{h}=0.10 ; F_{h}=0.88\right)$. Breaking down the oral absorption into specific components (i.e., absorption, gut, and hepatic extraction) allows a better understanding of the most influential factors driving oral bioavailability $\left(\mathrm{F}_{\text {oral }}\right)$. In addition, it gives an understanding of the first-pass drug burden to the GI tract and liver, which helps to develop improved physiologically based PK models to assist modeling of drug-drug interactions, for example. The low clearance and moderate oral bioavailability of nemiralisib (35\%) is regarded as suboptimal for inhaled drugs, which exert pharmacological action through first-pass topical delivery. Negligible oral bioavailability is generally preferred to minimize systemic toxicities from the swallowed portion of the dose (Cooper et al., 2012).

Following inhaled administration of nemiralisib, the time course of plasma concentrations of parent drug showed distinctly different profiles compared with oral dosing. The inhaled PK profiles showed rapid absorption, initial rapid distribution, and biexponential decline with marginally longer terminal phase elimination half-life ( $t_{1 / 2} 58.4$ hours) than obtained following oral and intravenous administration (50.8 and 54.7 hours, respectively). The inhaled absolute bioavailability of nemiralisib of $38 \%$ is likely to constitute drug derived via both an inhaled and swallowed component. Indeed, a previous study evaluating the absorption of nemiralisib through the lung by blocking absorption via the gut using charcoal indicated that $23 \%$ of the total systemic exposure after inhalation from the ELLIPTA device was attributable to orally absorbed drug (Wilson et al., 2018).

Parameters measured in this study can be pieced together into an understanding of the disposition of nemiralisib following inhalation administration. Such a scheme is presented in Fig. 8, which was constructed initially assuming inhaled and oral bioavailability of $38 \%$ and $35 \%$, respectively, with $23 \%$ of systemic exposure being derived from the swallowed component (Wilson et al., 2018). With this, the fraction of nemiralisib bioavailable via the lung $\left(\mathrm{F}_{\text {lung }}=\right.$ $29 \%$ ) and the swallowed proportion (25\%) of the total device dose can be estimated. Using this approach, there is a proportion of drug (approximately 46\%) that is unaccounted for. It is unclear why the calculation methods deployed in this work have resulted in such a low total accountability, although they compare well with values reported by Newman and Busse (2002), who quote dose emissions from the Rotahaler (GSK, Brentford, UK) Diskhaler (GSK, Brentford, UK), and Turbohaler (AstraZeneca, Cambridge, UK) dry powder inhalers to be in the range of $52 \%-63 \%$ of label claim and an $\mathrm{F}_{\text {lung }}$ range of $6.2 \%-40.5 \%$ across a range of different devices. An alternative approach to forming such a scheme, without reliance on in vivo charcoal block data, would be to predict the proportion of dose swallowed and deposited in the lung using in vitro aerodynamic particle size distribution data (Wilson et al., 2019).

Following intravenous and oral dosing, the major circulating drugrelated component in plasma was unchanged nemiralisib. A single radioactive peak representing approximately $8 \%-10 \%$ of sample radioactivity was composed of up to two metabolites resulting from $\mathrm{N}$-dealkylation and $\mathrm{N}$-dealkylation with sulfate conjugation. The proportion of individual metabolite, therefore, did not exceed the $10 \%$ threshold outlined by International Committee on Harmonization M3(R2) harmonized guidance (2009), above which additional qualification work in animals might be warranted. The chemical structures of the metabolites identified resulted from common 
oxidative and conjugative routes and are regarded as innocuous and unlikely to be associated with reactivity. M1 was approximately 16fold less potent against pi3Kd compared with nemiralisib (IC50 of 3.2 $\mathrm{nM}$ compared with $0.2 \mathrm{nM}$ - GSK unpublished data) and unlikely, therefore, to result in systemic toxicities at low inhalation dose levels $(<1 \mathrm{mg})$. The study has, therefore, not revealed any human nemiralisib metabolites needing further qualification in either invitro or further animal studies.

Unchanged nemiralisib was a major component in duodenal bile following intravenous administration but was a lesser component in feces extracts (Fig. 6), suggesting that enterohepatic recirculation of nemiralisib is a likely explanation for the secondary peaks observed in the PK plasma profiles and a contributing factor to the long terminal half-life and protracted elimination of the drug. Following oral administration, $24 \%$ of the administered dose was associated with unchanged parent in urine and feces (Table 6). Routes of metabolism included various oxidations including N-dealkylation and oxidation of the indole ring, sulfate conjugation, glucuronide conjugation, and combinations thereof. The renal blood clearance of nemiralisib was low $(0.93 \mathrm{l} / \mathrm{h})$, calculated using eq. 4 (Table 1$)$. Overall, these data indicate that nemiralisib and oxidative/conjugative metabolites are secreted into the bile to be excreted in feces. A high proportion of metabolic elimination is likely to result from enterohepatic recirculation of unchanged nemiralisib. Direct renal clearance and metabolism followed by renal clearance are lesser routes of elimination.

In conclusion, an innovative small two-part clinical pharmacology study employing concomitant dose routes, state-of-the-art technology and comprehensive estimations of ADME-PK parameters has enabled the inhaled disposition of nemiralisib to be rigorously characterized.

\section{Acknowledgments}

The authors would like to thank Bianca Squillaci who conducted the metabolite LCMS identification work, Steve Corless and Clive Felgate for their assistance with the AMS analysis, and Hazel Lawson-Harrison who contributed to sample pooling and extractions. Editorial support was provided by Kate Hollingworth of Continuous Improvement Ltd and funded by GSK.

\section{Authorship Contributions}

Participated in research design: Harrell, Wilson, Man, Riddell, Jarvis,

Young, Crossman, Kenworthy, Marotti, Wilkes, Hessel, Fahy.

Conducted experiments: Chambers, Crossman, Pereira, Wilkes.

Performed data analysis: Wilson, Man, Jarvis, Chambers, Crossman,

Kenworthy, Beaumont, Marotti, Fahy.

Wrote or contributed to the writing of the manuscript: All authors.

\section{References}

Affrime MB, Cuss F, Padhi D, Wirth M, Pai S, Clement RP, Lim J, Kantesaria B, Alton K, and Cayen MN (2000) Bioavailability and metabolism of mometasone furoate following administration by metered-dose and dry-powder inhalers in healthy human volunteers. $J$ Clin Pharmacol 40:1227-1236.

Ambery C, Young G, Fuller T, Lazaar AL, Pereira A, Hughes A, Ramsay D, van den Berg F, and Daley-Yates P (2018) Pharmacokinetics, excretion, and mass balance of [14 C]-batefenterol following a single microtracer intravenous dose (concomitant to an inhaled dose) or oral dose of batefenterol in healthy men. Clin Pharmacol Drug Dev 7:901-910.

Begg M, Wilson R, Hamblin JN, Montembault M, Green J, Deans A, Amour A, Worsley S, Fantom K, Cui Y, et al. (2019) Relationship between pharmacokinetics and pharmacodynamic responses in healthy smokers informs a once-daily dosing regimen for nemiralisib. J Pharmacol Exp Ther 369:337-344.

Bloomer JC, Nash M, Webb A, Miller BE, Lazaar AL, Beaumont C, and Guiney WJ (2013) Assessment of potential drug interactions by characterization of human drug metabolism pathways using non-invasive bile sampling. Br J Clin Pharmacol 75:488-496.

Cahn A, Hamblin JN, Begg M, Wilson R, Dunsire L, Sriskantharajah S, Montembault M, Leemereise CN, Galinanes-Garcia L, Watz H, et al. (2017) Safety, pharmacokinetics and doseresponse characteristics of GSK2269557, an inhaled PI3K $\delta$ inhibitor under development for the treatment of COPD. Pulm Pharmacol Ther 46:69-77.

Cooper AE, Ferguson D, and Grime K (2012) Optimisation of DMPK by the inhaled route: challenges and approaches. Curr Drug Metab 13:457-473.

Davies B and Morris T (1993) Physiological parameters in laboratory animals and humans. Pharm Res 10:1093-1095.

Down K, Amour A, Baldwin IR, Cooper AWJ, Deakin AM, Felton LM, Guntrip SB, Hardy C, Harrison ZA, Jones KL, et al. (2015) Optimization of novel indazoles as highly potent and selective inhibitors of phosphoinositide 3-kinase $\delta$ for the treatment of respiratory disease. $\mathrm{J}$ Med Chem 58:7381-7399.

Garner RC, Barker J, Flavell C, Garner JV, Whattam M, Young GC, Cussans N, Jezequel S, and Leong D (2000) A validation study comparing accelerator MS and liquid scintillation counting for analysis of 14C-labelled drugs in plasma, urine and faecal extracts. J Pharm Biomed Anal 24:197-209.

Guiney WJ, Beaumont C, Thomas SR, Robertson DC, McHugh SM, Koch A, and Richards D (2011) Use of Entero-Test, a simple approach for non-invasive clinical evaluation of the biliary disposition of drugs. Br J Clin Pharmacol 72:133-142

Harrell AW, Siederer SK, Bal J, Patel NH, Young GC, Felgate CC, Pearce SJ, Roberts AD, Beaumont C, Emmons AJ, et al. (2013) Metabolism and disposition of vilanterol, a long-acting $\beta(2)$-adrenoceptor agonist for inhalation use in humans. Drug Metab Dispos 41:89-100.

Hop CE, Wang Z, Chen Q, and Kwei G (1998) Plasma-pooling methods to increase throughput for in vivo pharmacokinetic screening. J Pharm Sci 87:901-903.

International Committee on Radiological Protection (1991) Recommendations of the international commission on radiological protection. ICRP Publication 60. Ann ICRP 21:1-3.

Keck BD, Ognibene T, and Vogel JS (2010) Analytical validation of accelerator mass spectrometry for pharmaceutical development. Bioanalysis 2:469-485.

Lappin G (2016) Approaches to intravenous clinical pharmacokinetics: recent developments with isotopic microtracers. J Clin Pharmacol 56:11-23.

Newman SP and Busse WW (2002) Evolution of dry powder inhaler design, formulation, and performance. Respir Med 96:293-304

Nomeir AA, Morrison R, Prelusky D, Korfmacher W, Broske L, Hesk D, McNamara P, and Mei H (2009) Estimation of the extent of oral absorption in animals from oral and intravenous pharmacokinetic data in drug discovery. J Pharm Sci 98:4027-4038.

Penner N, Klunk LJ, and Prakash C (2009) Human radiolabeled mass balance studies: objectives, utilities and limitations. Biopharm Drug Dispos 30:185-203.

Roffey SJ, Obach RS, Gedge JI, and Smith DA (2007) What is the objective of the mass balance study? A retrospective analysis of data in animal and human excretion studies employing radiolabeled drugs. Drug Metab Rev 39:17-43.

Wilson R, Jarvis E, Montembault M, Hamblin JN, Hessel EM, and Cahn A (2018) Safety, tolerability, and pharmacokinetics of single and repeat doses of nemiralisib administered via the Ellipta dry powder inhaler to healthy subjects. Clin Ther 40:1410-1417.

Wilson R, Templeton A, Leemereise C, Eames R, Banham-Hall E, Hessel EM, and Cahn A (2019) Safety, tolerability, and pharmacokinetics of a new formulation of nemiralisib administered via a dry powder inhaler to healthy individuals. Clin Ther 41:1214-1220.

Young GC, Corless S, Felgate CC, and Colthup PV (2008) Comparison of a $250 \mathrm{kV}$ single-stage accelerator mass spectrometer with a $5 \mathrm{MV}$ tandem accelerator mass spectrometer--fitness for purpose in bioanalysis. Rapid Commun Mass Spectrom 22:4035-4042.

Young GC, Seymour M, Dueker SR, Timmerman P, Arjomand A, and Nozawa K (2014) New frontiers-accelerator mass spectrometry (AMS): recommendation for best practices and harmonization from Global Bioanalysis Consortium Harmonization Team. AAPS J 16:357-359.

Address correspondence to: Andrew Harrell, Drug Metabolism and Pharmacokinetics, GlaxoSmithKline Research and Development, Ware, Hertfordshire SG12 ODP, United Kingdom. E-mail: andrew.w.harrell@gsk.com 